\title{
Analysis of a Late-Autumn Rainstorm in the Sichuan Basin on the Eastern Side of the Tibetan Plateau
}

\author{
Yongren Chen $\mathbb{D}^{1,2,3,4}$ and Yueqing $\mathrm{Li}^{1,3}$ \\ ${ }^{1}$ Institute of Plateau Meteorology, China Meteorological Administration, Chengdu, Sichuan 610072, China \\ ${ }^{2}$ Meteorological Disaster Defense Technology Center of Sichuan Province, Chengdu, Sichuan 610072, China \\ ${ }^{3}$ Heavy Rain and Drought-Flood Disasters in Plateau and Basin Key Laboratory of Sichuan Province, Chengdu 610072, China \\ ${ }^{4}$ Sichuan Provincial Meteorological Observatory, Chengdu, Sichuan 610072, China \\ Correspondence should be addressed to Yongren Chen; yr20060004@163.com
}

Received 6 January 2019; Revised 28 March 2019; Accepted 2 May 2019; Published 15 May 2019

Academic Editor: Helena A. Flocas

Copyright (c) 2019 Yongren Chen and Yueqing Li. This is an open access article distributed under the Creative Commons Attribution License, which permits unrestricted use, distribution, and reproduction in any medium, provided the original work is properly cited.

\begin{abstract}
An abnormal heavy rainfall that occurred on 27 October 2014 in the Sichuan Basin (SB), China, is analyzed. An inverted trough at $850 \mathrm{hPa}$ evolved into a Southwest China Vortex (SWCV), and strong upward motion caused by interaction between the low-level jet (LLJ) at $850 \mathrm{hPa}$ and the upper-level jet (ULJ) at $200 \mathrm{hPa}$ triggered the rainstorm process. Under a large-scale circulation system featuring a westerly trough and subtropical high, there were two cloud bands over the northeast side and south side of the Tibetan Plateau, respectively. Influenced by the eastward-moving trough, the inverted trough, LLJ, and the SWCV, a Mesoscale Convective System (MCS) was generated near the intersection of the two cloud bands, and it was the direct rainstorm system. The MCS strengthened under the situation of the $850 \mathrm{hPa}$ inverted trough, but weakened when the inverted trough evolved to into the SWCV. Eventually, it formed the phenomenon known as "existing vortex without cloud." Through analysis of the possible reasons why precipitation strengthened (weakened) under the situation of the inverted trough (SWCV), it was found that the strengthening of precipitation was due to a strong tilting updraft in the area of the ULJ and LLJ intersection. On one hand, the upward motion was related to the vorticity advection variation with height and the low-level warm advection forcing; while on the other hand, the dew-point front near the LLJ also played a lifting role in the upward flow of the lower-layer vertical circulation. Meanwhile, the LLJ "head" was a high-value area of water vapor convergence, which provided sufficient water vapor for the rainstorm. During the SWCV, the weakening of precipitation was due to the SWCV weakening gradually; plus, the ULJ was interrupted over the SB, the upper airflow presented downdrafts, and its superposition with the ascending branch of low-level vertical circulation. This airflow structure inhibited the development of strong upward motion, whilst at the same time, the LLJ retreated toward the south and the dew-point front ultimately weakened and disappeared. Subsequently, water vapor convergence weakened and no longer supported the occurrence of heavy rainfall. Therefore, the strong upward motion caused by the ULJ-LLJ intersection and the lower-level dew-point front were the key reasons for the occurrence of this late-autumn rainstorm.
\end{abstract}

\section{Introduction}

China is a country that experiences many rainstorms [1-4]. Among them, the rainstorms that occur in the upstream, steep-terrain region of the Yangtze River are notoriously difficult to understand and forecast and have long represented a difficult problem for meteorological research and operations relating to rainstorms in China $[5,6]$. The Sichuan Basin (SB), in Southwest China (Figure 1(a)), is on the leeward slope of the eastern side of the Tibetan Plateau and is characterized by distinct geomorphology such as steep terrain, mountain basins, lakes, and rivers. Moreover, it is the main path for the warm and wet southwesterly flow at low latitudes arriving in the eastern part of China. In summer, influenced by Tibetan Plateau weather systems (e.g., the Tibetan Plateau Vortex [7-11], Southwest China Vortex [12-15], and the Western Pacific Subtropical High (WPSH) [16]), not only there are many wide-ranging 


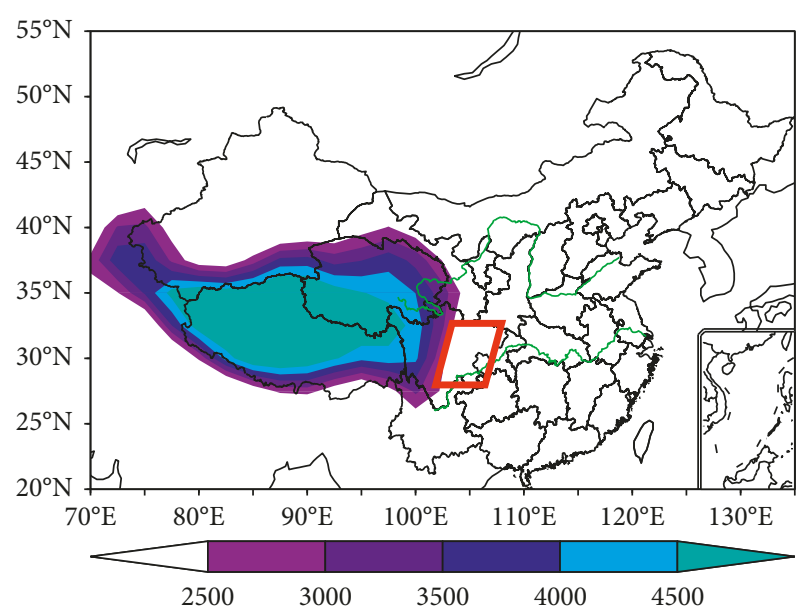

(a)

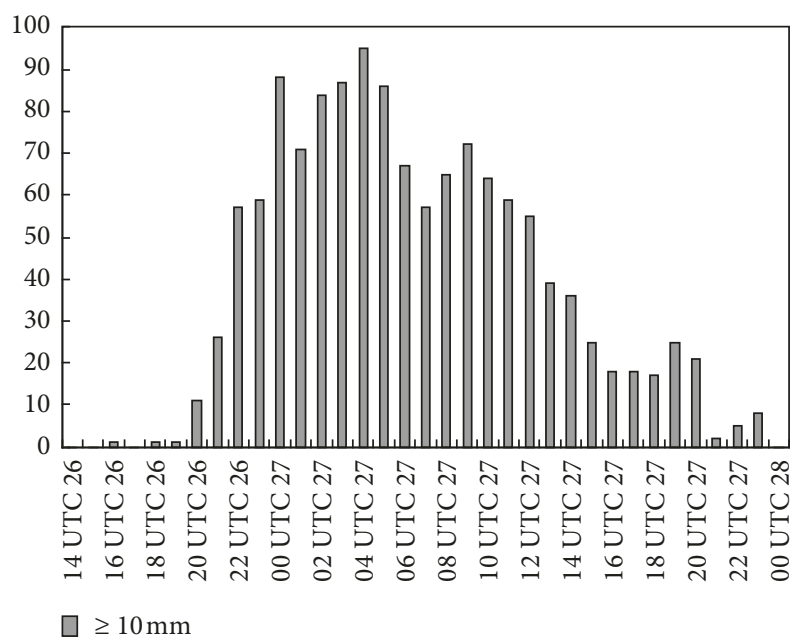

(c)

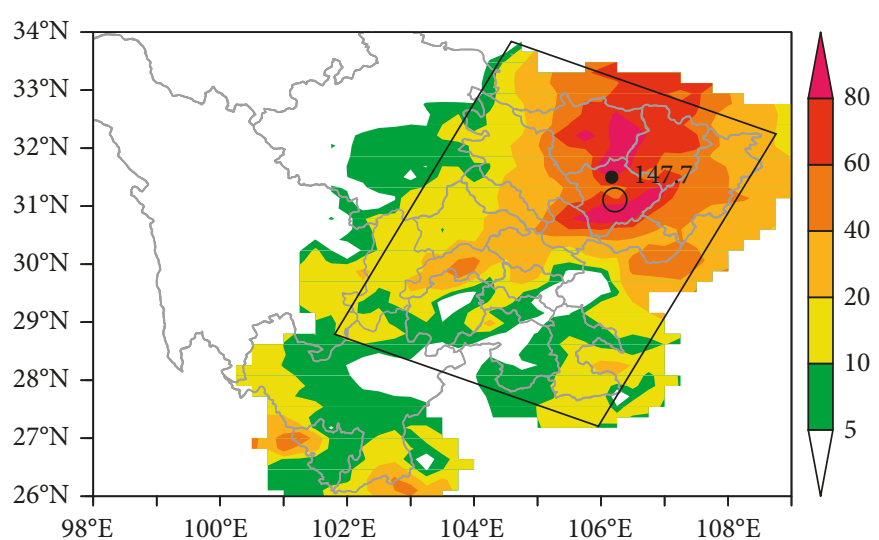

(b)

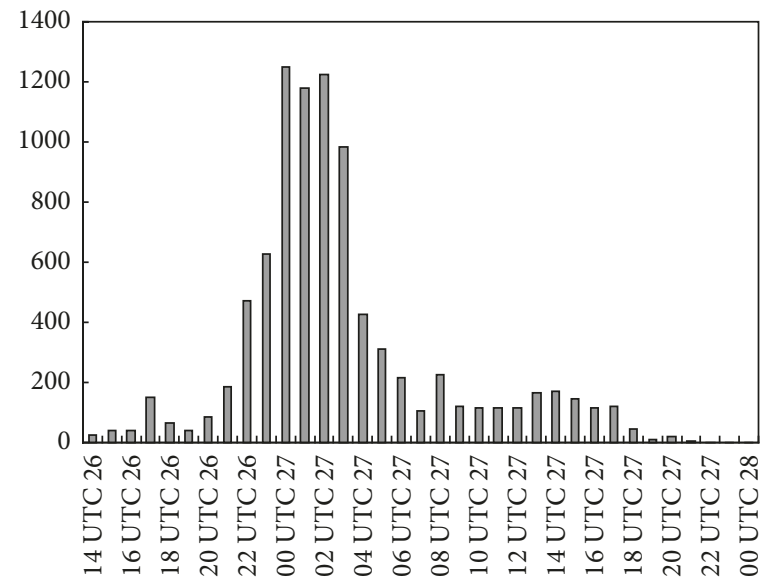

(d)

Figure 1: The location of Sichuan Basin (a) (the shaded area is terrain of Tibetan plateau (unit: $\mathrm{m}$ )), the accumulated precipitation (b) (in $\mathrm{mm}$ ) from 1200 UTC 26 to 0000 UTC 28 October 2014, the number of stations with hourly precipitation $\geq 10 \mathrm{~mm}$ (c), and the hourly lightning times (d). "•" is the location of precipitation center (Yilong); " $\bigcirc$ " indicates the location of the Nanchong radar.

and persistent heavy rainfall weather events, but also a large number of localized rainstorm processes occurring in the SB. These abnormally strong precipitation events, as well as the secondary disasters caused by such strong precipitation, often lead to substantial socioeconomic damage and losses. Therefore, as a typical area of China's rainstorm weather, the $\mathrm{SB}$ is known within the country for its high level of incidence regarding torrential rain and flooding.

Observational evidences have considerably demonstrated that the Tibetan Plateau Vortex, shear lines [17], and the Southwest China Vortex (SWCV) are the major weather systems responsible for rainstorms in Sichuan; plus, they have an important influence on the strong precipitation that occurs in the vast areas downstream of the Tibetan Plateau. The considerable research effort in recent decades in this regard has led to some meaningful achievements, such as on the generation sources of the Tibetan Plateau Vortex and SWCV in summer [18-20], the causes of their formation $[8,21-23]$, and their thermodynamic structure and effects on rainstorms [24, 25]. Recently, with the application of high-resolution data, the structure and organization of Mesoscale Convective Systems (MCSs) in these weather systems have been further revealed, indicating their importance in inducing the strong precipitation associated with such vortices $[12,24,26-29]$. On the other hand, because of the prominent effect that the East Asian summer monsoon has on the precipitation activity in China, most rainstorms in the SB occur in the months 6-9 of the warm season, with the frequency reducing significantly from October (i.e., the cold season), especially wide-ranging rainstorms. Therefore, existing research on rainstorms in Sichuan has mainly focused on summer, with little examination of rainstorms occurring in the winter half of the year.

In fact, the formation processes of a rainstorm are complex-for example, the large-scale conditions, the quantity of water vapor, the strong upward motion, and the 
level of instability in the atmospheric stratification, together with the effects of weather systems and topographies at multiple scales, all act in a synergistic way in the occurrence of severe rainstorm weather [30]. In addition, climate change has also been having an influence on the spatiotemporal distribution and evolutionary process of strong precipitation in many areas, including Sichuan [31-34]. Therefore, it is highly necessary and relevant to carry out research on abnormal rainstorm weather.

In the present study, an abnormal rainstorm that occurred in SB on 27 October 2014 was chosen for analysis. This late-autumn rainstorm had some convective weather features, such as a high frequency of lightning and a strong precipitation intensity. In general, the occurrence of high frequency of lightning activity and strong precipitation rates or high accumulated precipitation can not only be of convective results but also of baroclinic instability, as well, considering that such events occur during the warm period of the year; plus, it is very rare for such an event to be observed in the cold season of autumn, and in fact, it is the latest-occurring regional heavy rainfall event in recorded history (since 1955) in the Sichuan region. To reveal the causes of its abnormal occurrence, the study was carried out from the perspective of its impacting weather systems, convection triggering conditions, and physical formation processes. The broader aim was to deepen our understanding of cold-season rainstorm weather. Following this introduction, the paper is organized as follows. Section 2 describes the data and methods; Section 3 describes rainstorm weather background; Section 4 analyzes multiscale weather system features; Section 5 analyzes system configuration and physical structure over the rainstorm area; Section 6 explains favorable conditions for the occurrence of the heavy rainstorm; and Section 7 concludes the study.

\section{Data and Methods}

The data used in this study are as follows:

(1) Sounding observational data at 0000 UTC and 1200 UTC

(2) Global final (FNL) analysis data from the National Centers for Environmental Prediction (NCEP), with a spatial resolution of $1^{\circ} \times 1^{\circ}$ and temporal resolution of $6 \mathrm{~h}$ (hereafter referred to as NCEP_fnl; http://rda. ucar.edu/)

(3) Brightness temperature (Tb) data from the FY2D geostationary weather satellite provided by the $\mathrm{Na}$ tional Satellite Meteorological Center, China Meteorological Administration (http://www.nsmc.org.cn/ en/NSMC/Home/Index.html)

(4) The weather radar data including base reflectivity and $3 \mathrm{~km}$ height wind field of radar echo inversion and surface precipitation data

To analyze a relationship between rainstorm and the vertical ascending motion, quasigeostrophic approximation is used in the study, and we know the equation of $\omega[35,36]$ is

$$
\begin{aligned}
&\left(\nabla^{2}+\frac{f_{0}^{2}}{\bar{\sigma}_{\mathrm{s}}} \frac{\partial^{2}}{\partial p^{2}}\right) \omega= \frac{f_{0}}{\bar{\sigma}_{\mathrm{s}}} \frac{\partial}{\partial p}\left[\vec{V}_{g} \cdot \nabla\left(\xi_{g}+f\right)\right] \\
& \\
&+\underbrace{\left\{-\frac{1}{\bar{\sigma}_{\mathrm{s}}} \nabla^{2}\left[\vec{V}_{g} \cdot \nabla\left(\frac{\partial \Phi}{\partial p}\right)\right]\right\}}_{b},
\end{aligned}
$$

where $\nabla=(\partial / \partial x) i+(\partial / \partial x) j,\left(1 / \sigma_{s}\right)=$ const, and $\overline{\sigma_{s}}$ is static stability; $\omega$ is vertical velocity, $\xi_{g}$ is vorticity, $\vec{V}_{g}$ is wind vector, and $p$ is pressure; and $\Phi=f_{0} \psi, \Phi$ is geopotential height function, $f_{0}$ is constant value of $f$ (the Coriolis parameter), and $\psi$ is stream function.

The left side of equation (1) can be written as

$$
\left(\nabla^{2}+\frac{f^{2}}{\bar{\sigma}} \frac{\partial^{2}}{\partial p^{2}}\right) \omega \cong-\left[k^{2}+l^{2}+\frac{1}{\bar{\sigma}}\left(\frac{f_{0}^{2} \pi}{P_{00}}\right)^{2}\right] \omega,
$$

where $k$ is the wave number of $x$-direction and $l$ is the wave number of $y$ directions. Easily, formula (3) can be obtained:

$$
\omega \propto \frac{f_{0}}{\bar{\sigma}_{\mathrm{s}}} \frac{\partial}{\partial p}\left(-\vec{V}_{g} \cdot \nabla\left(\xi_{g}+f\right)\right)+\frac{1}{\bar{\sigma}_{\mathrm{s}}} \nabla^{2}\left[\vec{V}_{g} \cdot \nabla\left(\frac{\partial \phi}{\partial p}\right)\right] .
$$

For short-wave systems, the planetary geostrophic vorticity advection is secondary, meaning the a-item of equation (1) can be written as

$$
\frac{\partial}{\partial p}\left[-\vec{V}_{g} \cdot \nabla\left(\xi_{g}+f\right)\right] \approx \frac{\partial}{\partial p}\left(-\vec{V}_{g} \cdot \nabla \xi_{g}\right) .
$$

In addition, the b-item of equation (1) is the Laplacian of thickness (on temperature) advection, and we can get

$$
\frac{1}{\bar{\sigma}_{\mathrm{s}}} \nabla^{2}\left[\vec{V}_{g} \cdot \nabla\left(\frac{\partial \phi}{\partial p}\right)\right] \propto-\vec{V}_{g} \cdot \nabla\left(\frac{\partial \phi}{\partial p}\right) \propto \vec{V}_{g} \cdot \nabla \theta_{\mathrm{se}} .
$$

According to (1)-(5), expression (3) can be obtained as follows:

$$
\omega \propto \frac{f_{0}}{\bar{\sigma}_{\mathrm{s}}} \frac{\partial}{\partial p}\left(-\vec{V}_{g} \cdot \nabla \xi_{g}\right)+\vec{V}_{g} \cdot \nabla \theta_{\mathrm{se}}
$$

where $\theta_{\text {se }}$ is the pseudoequivalent potential temperature (PEPT), according to Davies-Jones' notes [37]; $\theta_{\text {se }}$ can be calculated as

$$
\theta_{\mathrm{se}}=T\left(\frac{1000}{p-e}\right)^{R_{d} / C_{\mathrm{pd}}} \exp \left(\frac{L \cdot r}{C_{\mathrm{pd}} T_{\mathrm{c}}}\right)
$$

where $r=0.6222(e /(p-e))$ is the water vapor mixing ratio, $\left(R_{d} / C_{\mathrm{pd}}\right)=0.2854, C_{\mathrm{pd}}=1005.7 \mathrm{~J} \cdot \mathrm{kg}^{-1} \cdot \mathrm{K}^{-1}, T_{\mathrm{c}}$ is the temperature of condensation height, $e$ is vapor pressure, $p$ is pressure, and $T$ is temperature. So, $\theta_{\text {se }}$ is a comprehensive physical quantity that includes temperature, humidity, and pressure and can reflect the varying characteristics of both moisture and temperature.

Formula (6) shows the vorticity advection and PEPT advection are the main factors impacting upon the vertical velocity. When the positive vorticity advection with height increases (or negative vorticity advection with height 
decreases), $(\partial / \partial p)\left(-\vec{V}_{g} \cdot \nabla \xi_{g}\right)<0$, and in the warm advection area $\left(-\vec{V}_{g} \cdot \nabla \theta_{\text {se }}>0\right) \omega<0$, conditions are conducive to a forced rise; otherwise, there will be a forced sink.

\section{Rainstorm Weather Background}

This rainstorm process that occurred in SB on 27 October 2014 was highly abnormal in terms of the strength of precipitation weather recorded in Sichuan stretching back to 1955. The main features were that it occurred very late in the year (in fact, the latest ever in recorded history in this area) and the range of the precipitation was large. According to the accumulated precipitation data available, there were 584 automatic weather stations that reached $50-100 \mathrm{~mm}, 55$ above $100 \mathrm{~mm}$, and the maximum precipitation was recorded at $147.7 \mathrm{~mm}$ (Figure 1(b)) in Yilong of SB. According to the evolution of hourly precipitation and hourly lighting activity, at 2300 UTC 26 to 0600 UTC 27 October 2014, the number of automatic weather stations recording a rainfall rate of $>10 \mathrm{~mm} / \mathrm{h}$ reached a peak and, correspondingly, so did the lightning activity (Figure 1(d)). Then, between 0600 UTC 27 and 2000 UTC 27 October 2014, this number reduced gradually, and again, correspondingly, so did the lightning activity. The data clearly show that lightning occurred mainly in the strong precipitation period and that the frequency reached its maximum at 0000 UTC 27 October 2014, indicative of an obvious thunderstorm with strong precipitation and convective weather characteristics; indeed, the range of the rainstorm and the number of lightning flashes were the largest ever recorded.

In short, observations showed that, in terms of occurrence time, scope, intensity, nature, and so on, this was an abnormal rainstorm case in SB.

\section{Multiscale Weather System Features}

4.1. Large-Scale Circulation and Systems of Influence. The occurrence of a rainstorm is often related to the interactions among different scale weather systems, and only under the stable control of large-scale weather systems such as a subtropical high [38], long-wave trough, shear line or largescale low-pressure system, direct precipitation systems can appear and produce strong precipitation. The rainstorm system of the present study (Figure 2) showed that, at 1200 UTC 26 October 2014, before the rainstorm began (Figure 2(a)), there was a westerly trough over the Tibetan Plateau on the northwest side of the $500 \mathrm{hPa}$ WPSH, and under its influence, there was a belt of cloud that appeared over the northern area of the Tibetan Plateau and converged with the cloud at the edge of the WPSH over Sichuan. Its Tb was -20 to $-50^{\circ} \mathrm{C}$. During the rainstorm, at 0000 UTC 27 October 2014 (Figure 2(b)), the westerly trough moved to the $\mathrm{SB}$ and a new trough formed near $95^{\circ} \mathrm{E}$ in the south of the Tibetan Plateau. Also, the two cloud belts intersected over the $\mathrm{SB}$ area. At $850 \mathrm{hPa}$, the wind situation is one of northeasterly winds in the western $\mathrm{SB}$ and southeasterly winds in the eastern SB; this cyclonic flow field formed a " $\wedge$ " pattern (also known as an inverted trough) [26]. Under the trough's influence, an MCS with $\mathrm{Tb}$ values in the cloud of less than $-40^{\circ} \mathrm{C}$ was in the rainstorm area. By 1200 UTC 27 October 2014, the MCS was in the eastern part of the SB (Figure 2(c)), the $850 \mathrm{hPa}$ inverted trough had become a low vortex circulation (i.e., SWCV), and the low-level jet (a strong wind belt, which has a center wind speed of $\geq 12 \mathrm{~m} \cdot \mathrm{s}^{-1}$ in lower level of atmosphere $[3,39,40])$ and westerly trough were still located in the rainstorm area. At 0000 UTC 28 October 2014, the $500 \mathrm{hPa}$ westerly trough moved eastward out of Sichuan, the LLJ weakened, the convective systems in the large-scale cloud system were gradually removed from the SB, but the $850 \mathrm{hPa}$ SWCV remained over SB. As seen from the circulation situation, against the background of the westerly trough and WPSH, two cloud belts appeared on the northeast and south side of the Tibetan Plateau, respectively. Because of the influence of the trough, LLJ, and the vortex, an MCS with low Tb values formed in the area of the two cloud bands' intersection, which was a direct system of influence for the rainstorm weather. According to the $2 \mathrm{~h} \mathrm{~Tb}$ evolution (Figure 3), in the early stage of the $850 \mathrm{hPa}$ inverted trough's formation, the cloud at the south side of the Tibetan Plateau had affected the Sichuan area, but the cloud at the northeast side of it had not.

At 1200 UTC 26 October 2014, the southerly wind became weak, there was no obvious weather system at the low level, and the $\mathrm{Tb}$ value remained high (Figures 3(a)3(d)). At 0000 UTC 27 October 2014, under the influence of the inverted trough, there was an LLJ forming in the rainstorm area, and the Tb value of the MCS had reduced (Figures 3(e)-3(g)). During the inverted trough's evolution toward the SWCV (Figures $3(\mathrm{~h})-3(\mathrm{k})$ ), the MCS became slightly weakened and lasted about $8 \mathrm{~h}$. During the SWCV's existence (Figures 3(l)-3(p)), the MCS was located from the right-hand side of Chongqing to western Hubei, and SB featured no obvious cloud belt and the rainstorm ended.

The above shows that when the $850 \mathrm{hPa}$ inverted trough evolved toward the SWCV, this severe rainstorm event over SB occurred; however, once the SWCV had formed, the precipitation over SB weakened. So, the $850 \mathrm{hPa}$ inverted trough and the midtropospheric trough were the main systems of influence for this rainstorm event. Meanwhile, the MCS was a system that directly induced the rainstorm.

4.2. Mesoscale Weather Systems. Using the wind field of the Nanchong radar inversion at $3 \mathrm{~km}$ height, the MCS structure and mesoscale weather systems (omitted) were analyzed. The $6 \mathrm{~min}$ interval echo evolution of $0.5^{\circ}, 1.5^{\circ}$, and $2.4^{\circ}$ showed that convective systems existed in the rainstorm area, with the echo intensity reaching $35-50 \mathrm{dBZ}$. In the same area, the strong echo duration barely exceeded $1 \mathrm{~h}$, mostly displaying a "strengthening-weakening-strengthening" pattern of variation.

Figure 4 shows the echo reflectivity for the inverted trough and SWCV at a time, from which it can be seen that, for the inverted trough situation (Figures 4(a)-4(c)), the echo of $35-50 \mathrm{dBZ}$ was mainly located to the west of $107^{\circ} \mathrm{E}$ and north of $30^{\circ} \mathrm{N}$. The strong echoes in area A were 


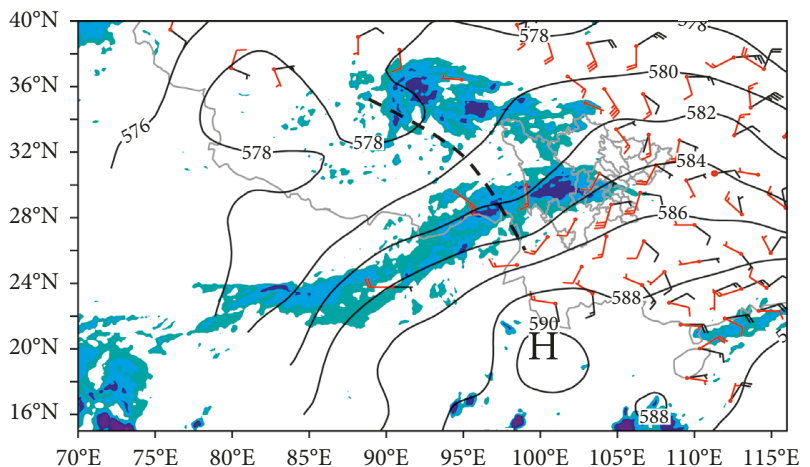

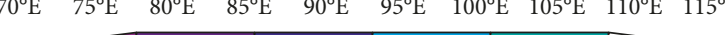
$-60$

(a)

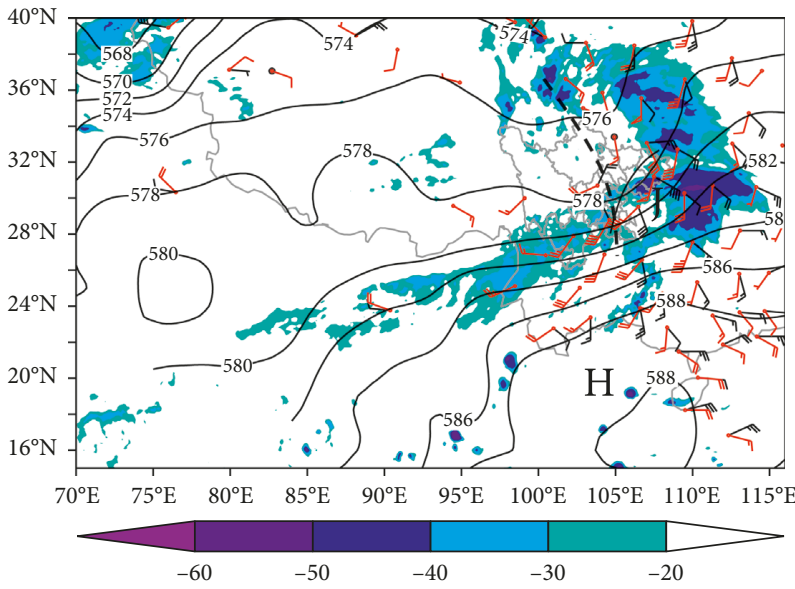

(c)

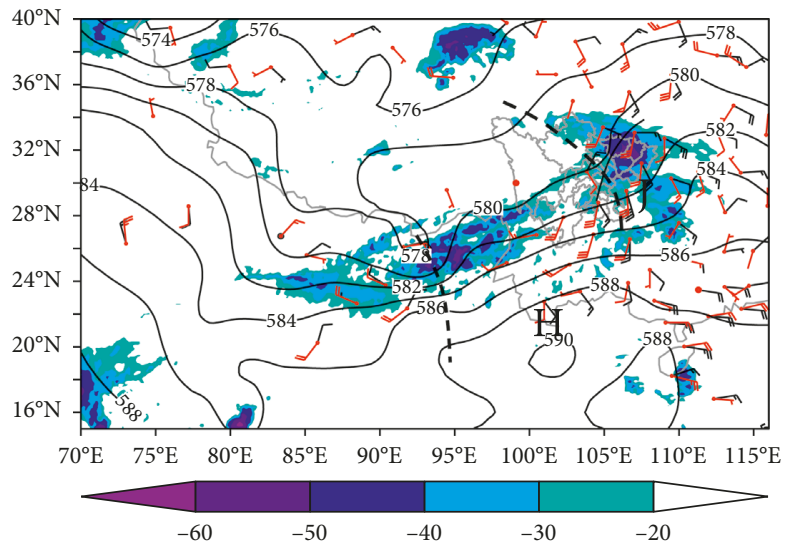

(b)
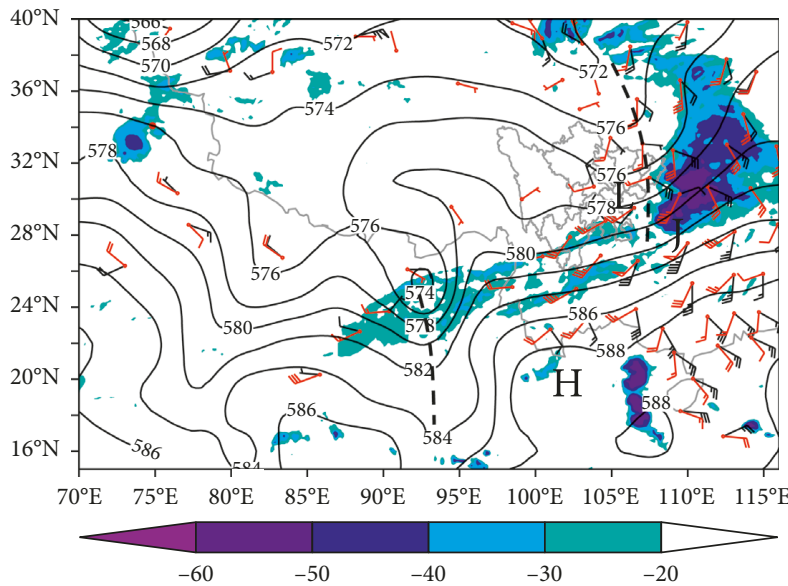

(d)

FigURE 2: $500 \mathrm{hPa}$ geopotential height (gpdam) and wind fields (red, $\mathrm{m} \cdot \mathrm{s}^{-1}$ ), $850 \mathrm{hPa}$ wind field (black, $\mathrm{m} \cdot \mathrm{s}^{-1} ; \mathrm{LLJ}: \geq 12 \mathrm{~m} \cdot \mathrm{s}^{-1}$ ), and Tb distribution (shaded, ${ }^{\circ} \mathrm{C}$ ) at (a) 1200 UTC 26 October 2014, (b) 0000 UTC 27 October 2014, (c) 1200 UTC 27 October 2014, and (d) 0000 UTC 28 October 2014. H-WPSH; L-SWCV; J-LLJ; wide broken line- $500 \mathrm{hPa}$ trough; wide solid line $-850 \mathrm{hPa}$ inverted trough.

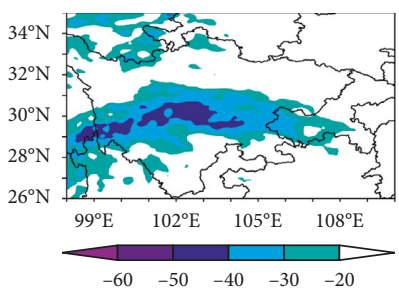

(a)

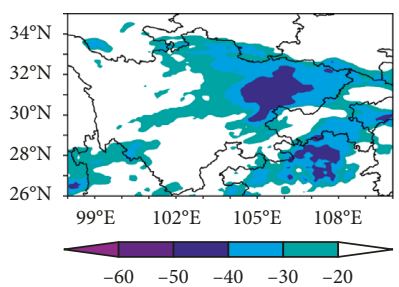

(e)

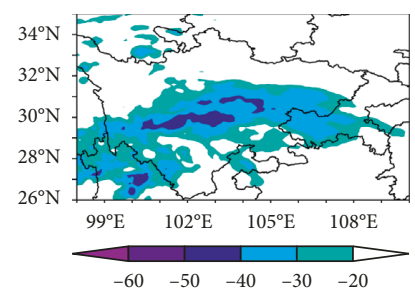

(b)

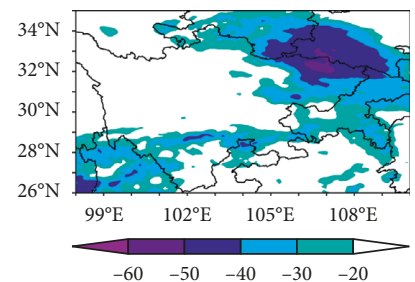

(f)

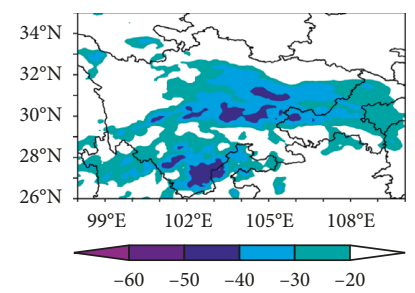

(c)

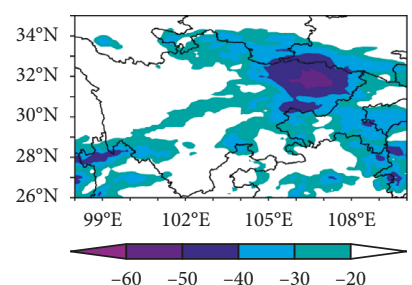

(g)

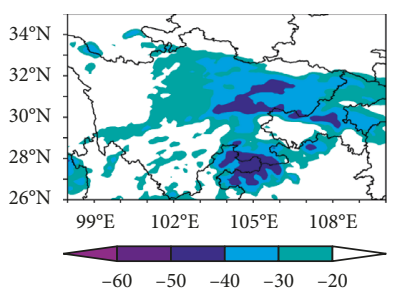

(d)

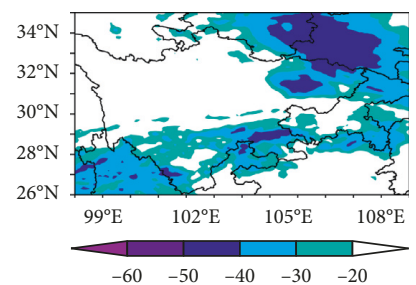

(h)

Figure 3: Continued. 


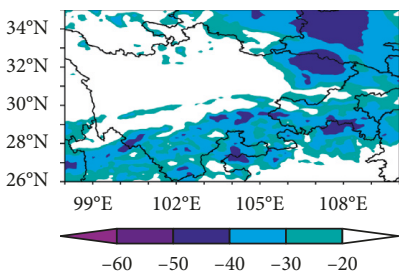

(i)

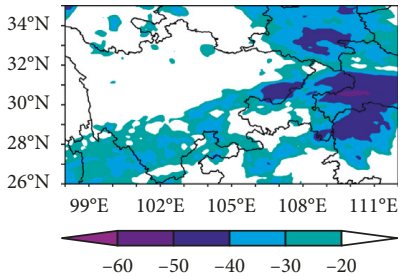

$(\mathrm{m})$

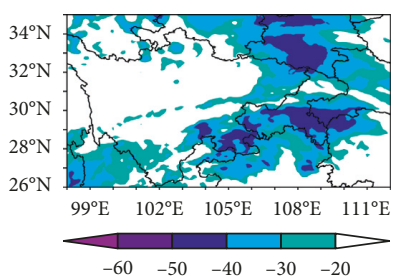

(j)

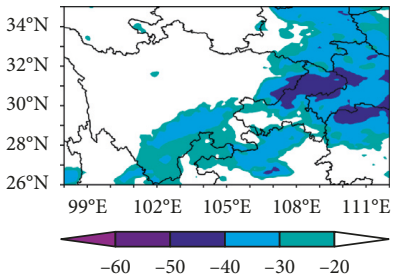

(n)

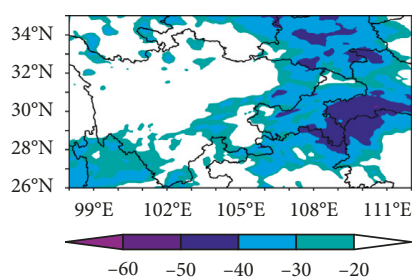

(k)

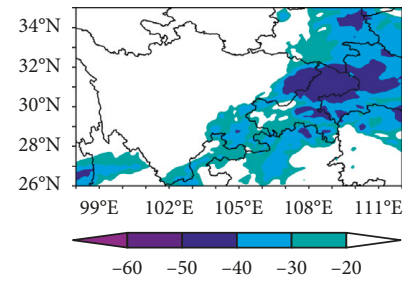

(o)

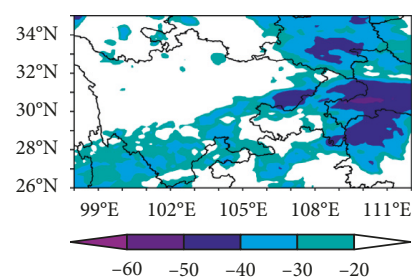

(1)

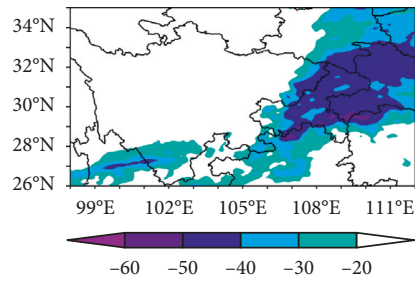

(p)

Figure 3: Tb distribution every $2 \mathrm{~h}$ from 1400 UTC 26 October to 2000 UTC 27 October 2014 (shaded, ${ }^{\circ} \mathrm{C}$ ). (a) 1400 UTC 26 , (b) 1600 UTC 26, (c) 1800 UTC 26, (d) 2000 UTC 26, (e) 2200 UTC 26, (f) 0000 UTC 27, (g) 0200 UTC 27, (h) 0400 UTC 27, (i) 0600 UTC 27, (j) 0800 UTC 27, (k) 1000 UTC 27, (l) 1200 UTC 27, (m) 1400 UTC 27, (n) 1600 UTC 27, (o) 1800 UTC 27, (p) 2000 UTC 27.

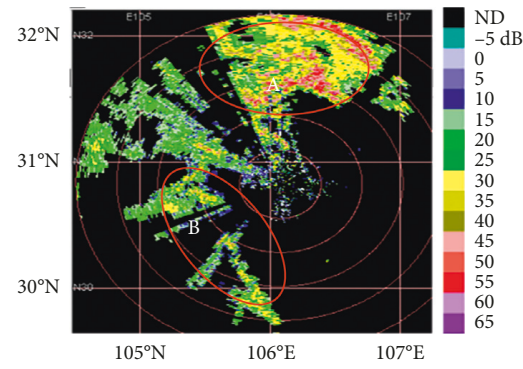

(a)

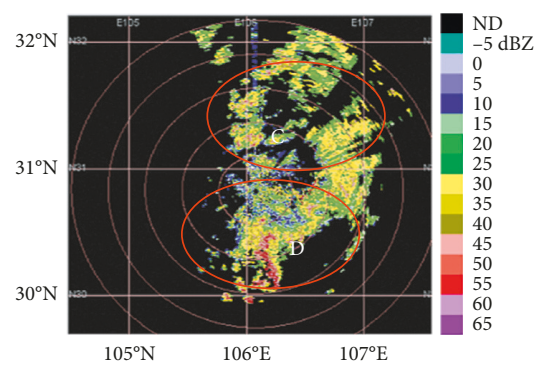

(d)

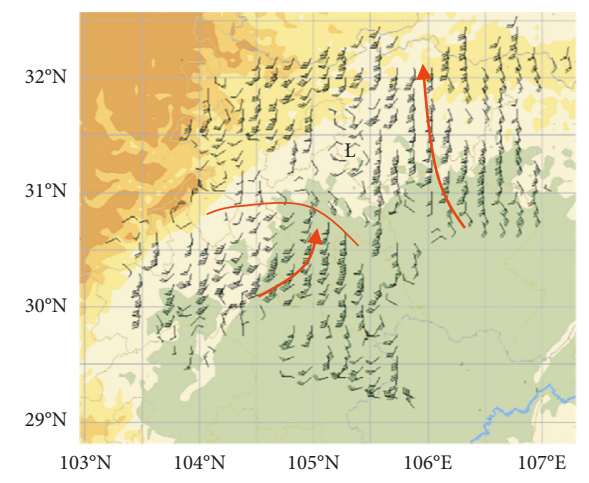

(g)

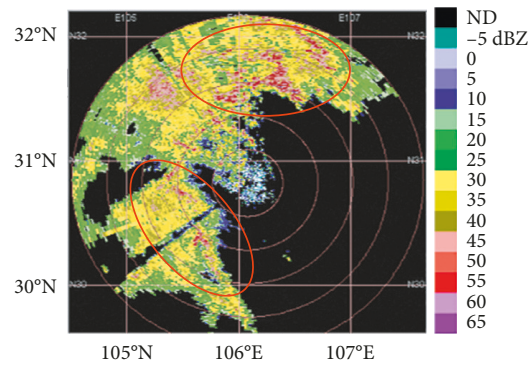

(b)

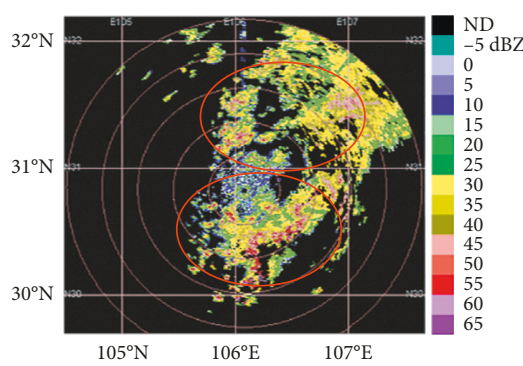

(e)

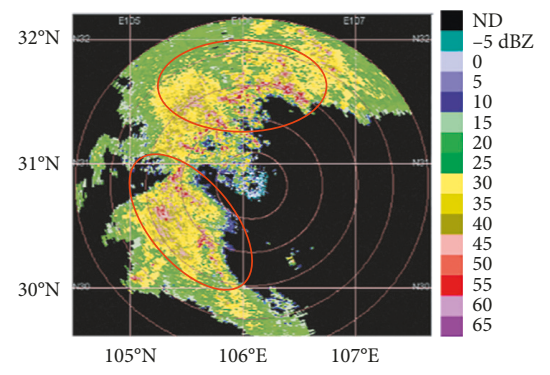

(c)

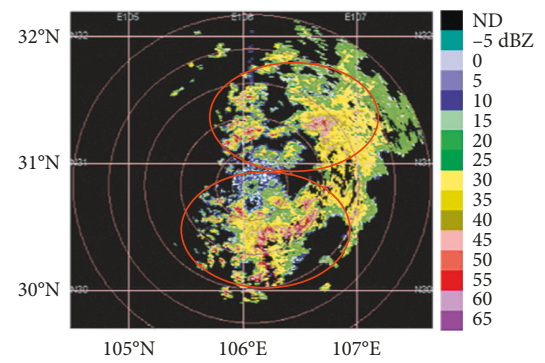

(f)

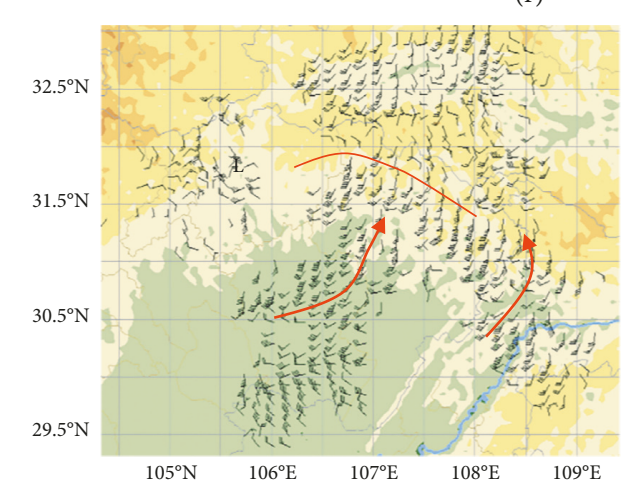

(h)

Figure 4: Base reflectivity of Nanchong radar $(\mathrm{dBZ})$ and corresponding $3 \mathrm{~km}$ height wind field of radar echo inversion $(\mathrm{g}$ and $\mathrm{h})\left(\mathrm{m} \cdot \mathrm{s}^{-1}\right)$ at (a-c, and g) 0105 UTC 27 October 2014, (d-f, and h) 1157 UTC 27 October 2014 (solid line-shear line; L-low vortex; solid-line arrows-small-scale LLJ) at the elevation of $0.5^{\circ}(\mathrm{a}, \mathrm{d}), 1.5^{\circ}(\mathrm{b}, \mathrm{e})$, and $2.4^{\circ}(\mathrm{c}, \mathrm{f})$. 
observable at all three elevations, whereas in area $\mathrm{B}$, they were mainly concentrated at $1.5^{\circ}$ and $2.4^{\circ}$. This indicated that the echo to the north of the radar station was deeper; and from the 1-hour precipitation evolution (omitted), the rainstorm intensity reached more than $20 \mathrm{~mm} / \mathrm{h}$ in deepecho area, mainly occurring at the rear of the inverted trough and in the northwestern SB near the trough line, and the range of strong precipitation was also large. For the SWCV situation (Figures $4(\mathrm{~d})-4(\mathrm{f})$ ), the echo values of 35-50 dBZ were mainly east of $106^{\circ} \mathrm{E}$. The strong echo had mid- $\gamma$-scale characteristics (zones C and D) and was sufficiently deep to be observed by radar at different elevations. Correspondingly, the strong precipitation mainly occurred on the righthand side of the SWCV. In the strong echo, the radar retrieval wind field at $3 \mathrm{~km}$ height showed that small-scale systems, such as the LLJ, vortices, and shear lines remained in the MCS (Figures 4(g) and 4(h)). The horizontal scale of these systems was about $1-50 \mathrm{~km}$, and the precipitation caused by them was stronger compared to the precipitation around this area. For instance, on two occasions near the small-scale vortices, the $1 \mathrm{~h}$ precipitation in the vortex center was greater than $10 \mathrm{~mm}$, with the maximum reaching $15.5 \mathrm{~mm}$ and $11.3 \mathrm{~mm}$, respectively. For the shear line and LLJ, because their horizontal scales were larger than the small-scale vortices, the precipitation range of $>10 \mathrm{~mm} / \mathrm{h}$ was also larger.

Clearly, the vortex, trough, and shear line existed as different scale disturbances due to the different resolution of the observational data, and weather systems of all scales resulted in the occurrence of the rainstorm via their interaction. To a large extent, the configuration of large-scale and medium-scale weather systems had the major influence on the distribution of the precipitation, while the small-scale systems mainly affected the local precipitation area and intensity. Therefore, within the large-scale circulation, there were still smaller-scale systems in the MCS, which is reflective of the multiscale characteristics of this severe rainstorm.

The next step, therefore, was to investigate how, under the influence of the weather systems of various scales, the strong rainstorm enhanced during the evolution from the inverted trough into the vortex and then weakened in the period of the vortex until the phenomenon known as "existing vortex without cloud" appeared. Below, the vertical circulation distribution and variation in physical quantities in the rainstorm area are further analyzed.

\section{System Configuration and Physical Structure over the Rainstorm Area}

Besides the condition of abundant water vapor, the formation of a rainstorm also depends on strong upward motion, which is closely related with the atmospheric thermal and dynamic conditions and other externally forced conditions. Therefore, analysis of the thermodynamic effects over the rainstorm area was necessary. Against the complex terrain background in Sichuan, routine observational data are sparse, which often makes it difficult to describe the inverted trough, SWCV, and MCS structure in the SB region. So, using relatively high-resolution data that can better reflect the weather systems at play is very important.

Here, based on NCEP_FNL data, Figure 5 shows the $850 \mathrm{hPa}$ wind field and physical quantity distributions during the evolution from the inverted trough toward the SWCV. The shading is the $24 \mathrm{~h}$ variable temperature, the contours lines are values of the PEPT, and the dashed lines are the divergence. In the early stage of the $850 \mathrm{hPa}$ inverted trough (Figure 5(a)), the low-level wind over the SB was convergent but the wind speed was weak $(4-6 \mathrm{~m} / \mathrm{s})$. The area north of $32^{\circ} \mathrm{N}$ featured significantly negative temperature variation (below $-2^{\circ} \mathrm{C}$ ), and the PEPT was $52-56^{\circ} \mathrm{C}$. When the inverted trough formed (Figure 5(b)), southeasterly wind over the rainstorm area strengthened and the maximum wind speed reached $16 \mathrm{~m} / \mathrm{s}$. Also, there was a positive temperature variation of $2-4^{\circ} \mathrm{C}$ in the central wind speed area, the wind convergence became stronger (maximum: $-6 \times 10^{-5} \mathrm{~s}^{-1}$ ), and the PEPT was $56-60^{\circ} \mathrm{C}$. The negative temperature variation in the northern SB expanded toward the south, and the thermodynamic conditions became conducive to the occurrence of strong precipitation.

When the SWCV formed (Figure 5(c)), the LLJ on the right-hand side of the SWCV was still present, low-level convergence enhanced slightly (maximum: $-8 \times 10^{-5} \mathrm{~s}^{-1}$ ), the PEPT was $56-60^{\circ} \mathrm{C}$, and the negative temperature variation began to affect the northern part of the SWCV. When the vortex circulation weakened (Figure 5(d)), the negative temperature variation had invaded the SWCV center, the PEPT was $50-55^{\circ} \mathrm{C}$, the LLJ on the right-hand side of the vortex weakened and moved toward the south, and the rainstorm weather over SB ended gradually.

It is clear that during the strengthening of precipitation, the low-level wind field always remained as an LLJ, there was positive temperature variation in the LLJ area, and the wind convergence significantly enhanced compared to the approaching rainstorm. Correspondingly, the number of stations with $>10 \mathrm{~mm} / \mathrm{h}$ and frequent hourly lightning flashes reached a peak. During the weakening of the rainstorm, the LLJ moved toward the south of the SB, negative temperature variation invaded the SWCV center, and the positive temperature variation weakened near the LLJ.

The configuration of the upper-level jet (ULJ) [41] and LLJ (Figure 6) was that during the conversion of the inverted trough into the SWCV (Figures 6(a)-6(c)), the $200 \mathrm{hPa}$ ULJ was always located over the south of Sichuan and the $850 \mathrm{hPa}$ LLJ expanded gradually, with both intersecting in the SB area. Also, there was a positive vorticity belt on the north side of the ULJ (central value of $9 \times 10^{-5} \mathrm{~s}^{-1}$ ), the left-hand side of the LLJ was also a positive vorticity center, and the MCS could develop near the intersection of the two jets. During the weakening of the vortex circulation (Figure 6(d)), the ULJ appeared to break over the SB, the LLJ retreated toward the south, the ULJ and LLJ no longer intersected and, correspondingly, the MCS weakened.

Past study [42] showed that interaction between upper and lower tropospheric jet streaks is an important factor in the development of organized severe convective storm systems. In this case, why when the ULJ and LLJ intersected over the SB was it conducive to the activities of the MCS and 


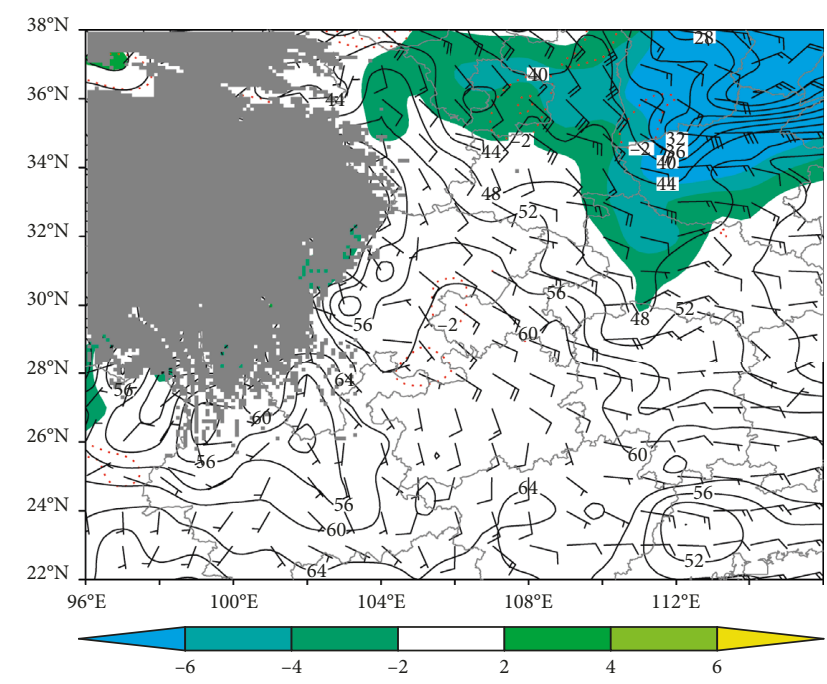

(a)

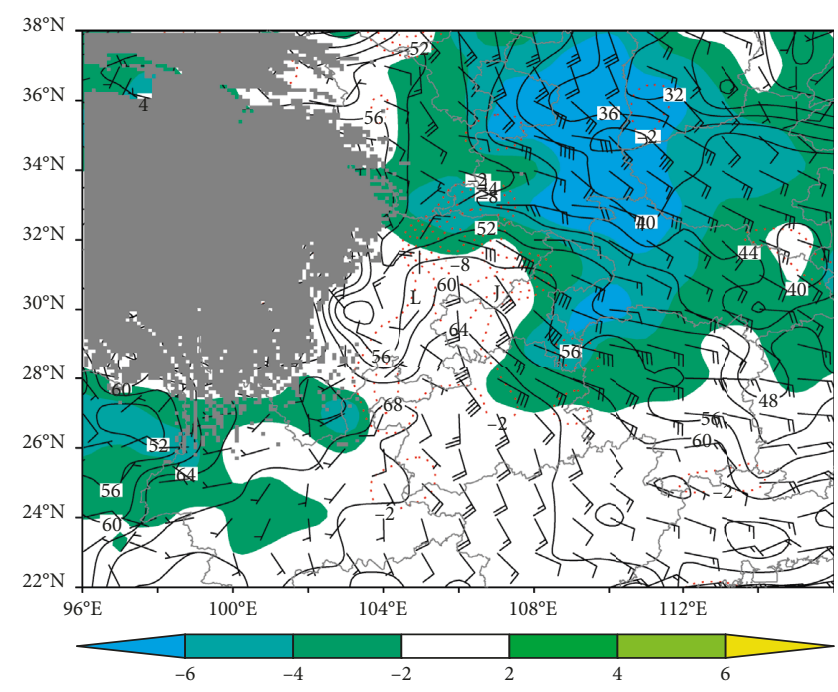

(c)

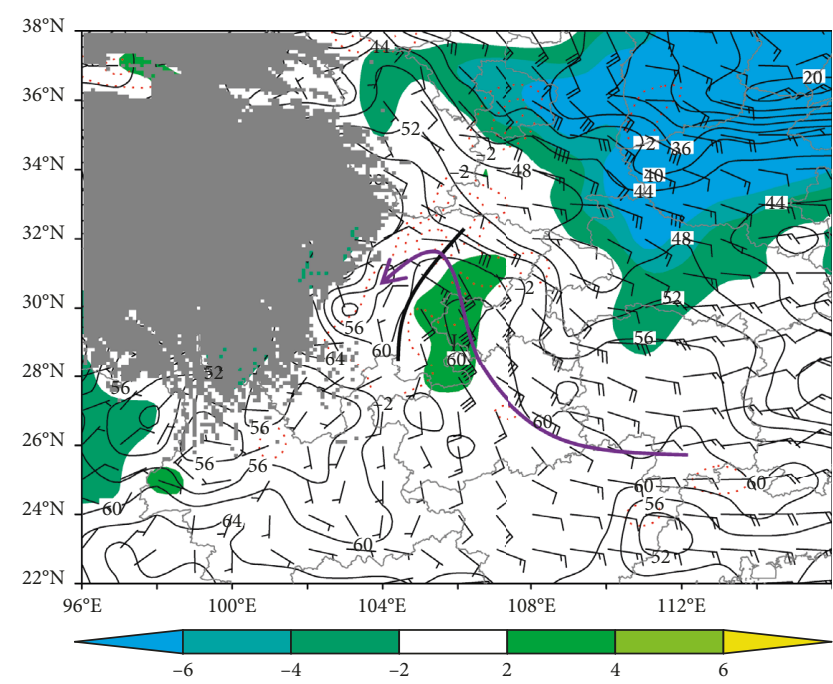

(b)

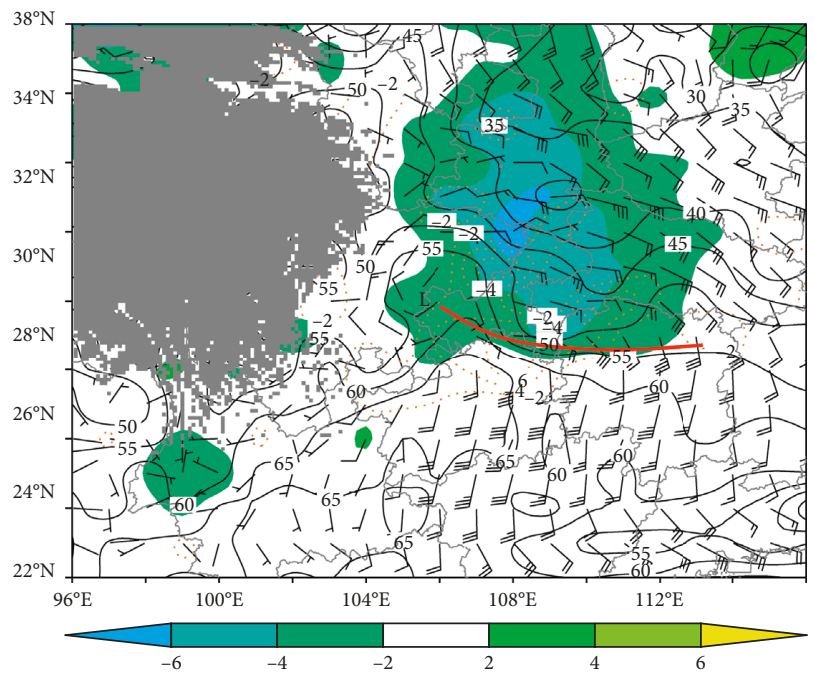

(d)

FiguRe 5: $850 \mathrm{hPa}$ wind field $\left(\mathrm{m} \cdot \mathrm{s}^{-1}\right), 24$-h temperature variation (shaded, ${ }^{\circ} \mathrm{C}$ ), divergence value of less than $-2 \times 10^{-5} \mathrm{~s}^{-1}$ (color dashed line), and PEPT distribution (black solid line, ${ }^{\circ} \mathrm{C}$ ) at (a) 1800 UTC 26 October 2014, (b) 0000 UTC 27 October 2014, (c) 1200 UTC 27 October 2014, and (d) 0000 UTC 28 October 2014. L-SWCV; J-LLJ. The thick solid line (black) and the curve with an arrow in (b) -inverted trough and its airflow direction; the thick solid line (red) in (d)-shear line; grey area-terrain of Tibetan Plateau.

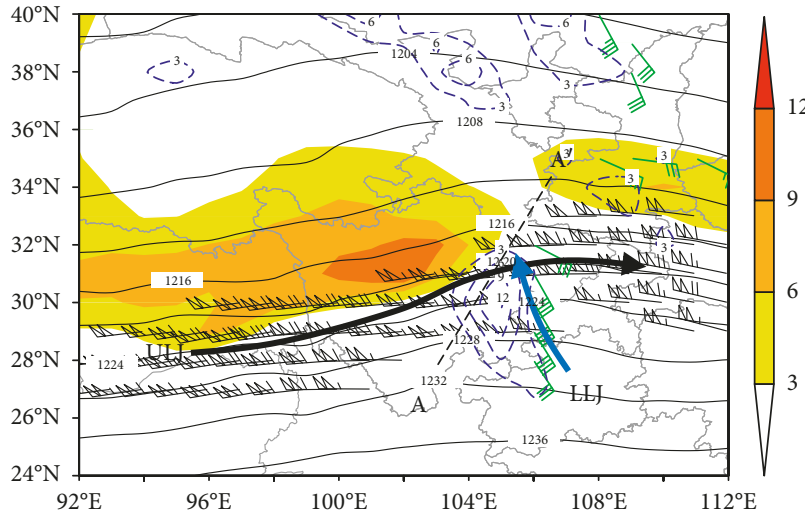

(a)

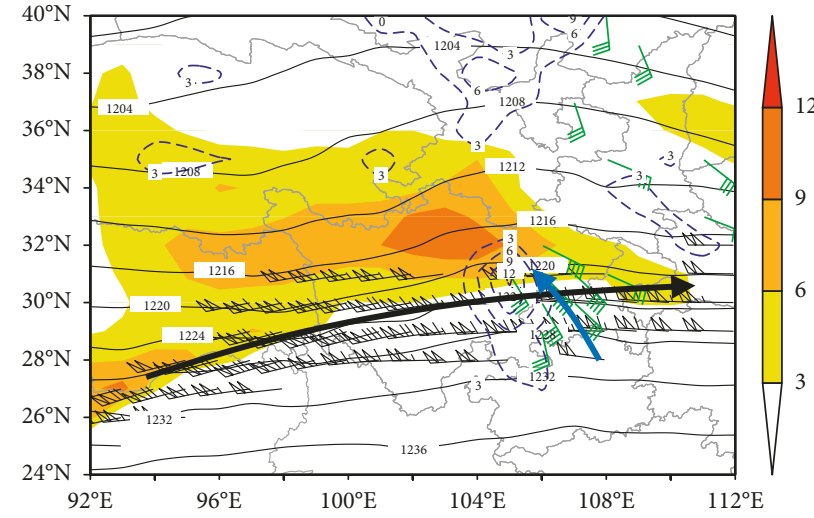

(b)

FIgUre 6: Continued. 


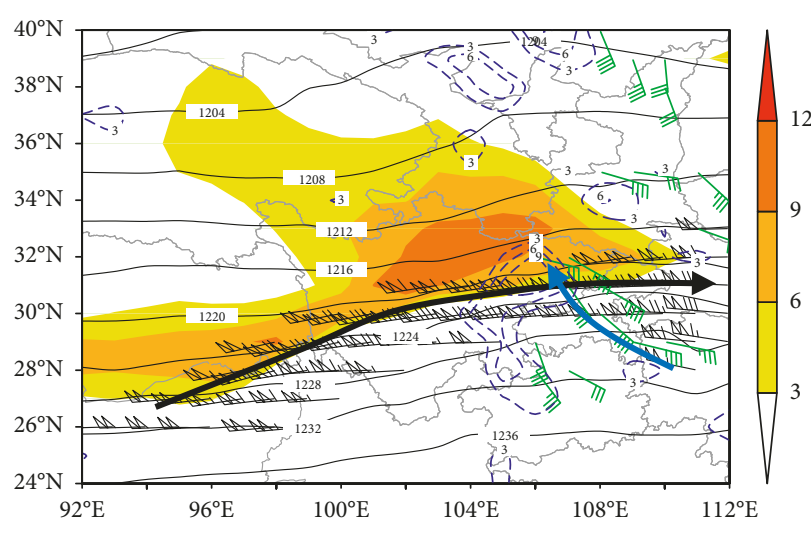

(c)

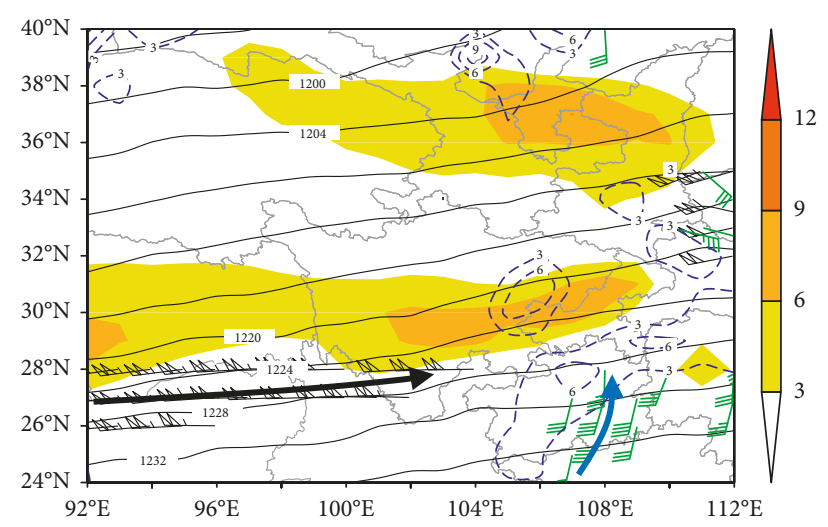

(d)

Figure 6: $200 \mathrm{hPa}$ ULJ (black wind vectors, $\mathrm{m} \cdot \mathrm{s}^{-1}$ ), geopotential height (solid line, gpdam), and vorticity (shading, $10^{-5} \mathrm{~s}^{-1}$ ), and $850 \mathrm{hPa}$ LLJ (green wind vectors, $\mathrm{m} \cdot \mathrm{s}^{-1}$ ) and vorticity (dotted line, $10^{-5} \mathrm{~s}^{-1}$ ), at (a) 0000 UTC 27 October 2014, (b) 0600 UTC 27 October 2014, (c) 1200 UTC 27 October 2014, and (d) 0000 UTC 28 October 2014. Black thick solid line-ULJ axis, blue thick solid line-LLJ axis.

the induction of the rainstorm, when the two jets did not intersect the MCS and its precipitation weakened? Taking a vertical section along the line A- $\mathrm{A}^{\prime}$ in Figure 6(a), the vertical circulation near the jets (Figure 7) was further analyzed. It can be seen that when the ULJ and LLJ intersected during the rainstorm (Figures $7(\mathrm{a})-7(\mathrm{c})$ ), the ascending branch of vertical circulation at the low level of the SB and the ascending branch below the ULJ axis superimposed upon each other, and this superposition effect promoted the formation of deep upward motion (values of less than $-1.0 \times 10^{-2} \mathrm{~Pa} / \mathrm{s}$ ). In addition, the two airflows were in an inclined upward state, which can keep the vertical wind shear from strengthening too much and thus generate a condition that is more conducive to the occurrence of heavy rainfall.

During the end of the rainstorm (Figure 7(d)), the two jets no longer intersected, the ascending branch of the vertical circulation at the low level of the SB and the descending branch below the ULJ axis superimposed upon each other, and this configuration suppressed the formation of strong upward motion such that conditions were not conducive to the development of convection. Therefore, although the vortex circulation was still intact at 0000 UTC 28 October 2014, the ULJ over the SB had broken, and the accompanying descending-branch airflow and ascending branch of secondary circulation at the low level of the SB were superimposed upon each other, which inhibited the occurrence of strong precipitation; this was one of the key reasons for the formation of having vortex but with no precipitation cloud in the SB. Therefore, the intersection of the ULJ and LLJ is an important configuration for inducing convection. Once the convection triggered by the ascending branch of secondary circulation develops, it often results in a severe rainstorm. Meanwhile, the LLJ was strengthened or maintained by latent heat release and vertical transport, and this was also why the LLJ horizontal scale was smaller under the situation of the inverted trough but expanded under that of the SWCV.

Further analyzing the characteristics of vorticity and divergence near the jets, Figure 8 shows that during the period when the inverted trough evolved toward the SWCV, the vertical structure of the lower-level (upper-level) convergence (divergence) (LLC and ULD, respectively) maintained, and the positive vorticity gradually strengthened in the midupper level. During the period of the SWCV weakening, the LLC-ULD structure disappeared and the low-level positive vorticity weakened. Specifically, at 0000 UTC 27 October 2014 (Figure 8(a)), the positive vorticity had an inclining distribution from the ground to $200 \mathrm{hPa}$, with the values in the lower level and upper level reaching $6 \times 10^{-5} \mathrm{~s}^{-1}$ and $1-3 \times 10^{-5} \mathrm{~s}^{-1}$, respectively, and this formed a vertical structure of the LLC-ULD in which convergence reached $-4 \times 10^{-5} \mathrm{~s}^{-1}$. During 0600-1200 UTC 27 October 2014 (Figures 8(b) and 8(c)), the LLC-ULD structure still maintained and the positive vorticity further increased at the midupper level (values $>9 \times 10^{-5} \mathrm{~s}^{-1}$ ). This physical structure was conducive to the formation of a pumping effect, which enhanced water vapor convergence at the low level and induced the development of a low-pressure system. When the SWCV weakened at 0000 UTC 28 October 2014 (Figure $8(\mathrm{~d})$ ), the LLC-ULD structure disappeared and the positive vorticity weakened at the low level, which was not conducive to the development of the pumping effect and low-pressure system. Thus, the dynamic variation near the jets involved positive vorticity strengthening at the midupper level and maintaining the LLC-ULD vertical structure during the strong precipitation, whereas during the period when the precipitation weakened, the positive vorticity reduced significantly at the low level and the LLC-ULD structure tended to disappear; thus, the dynamic conditions of the rainstorm's occurrence varied obviously.

Regarding the thermodynamic conditions and considering the importance of the PEPT for the occurrence of the rainstorm, the $24 \mathrm{~h}$ variation in PEPT was calculated by $\Delta_{24 h} \theta_{\mathrm{se}}=\theta_{\mathrm{se}_{2}}-\theta_{\mathrm{se}_{1}}$, where $\theta_{\mathrm{se}_{2}}$ is the PEPT at the current time and $\theta_{\mathrm{se}_{1}}$ is the PEPT $24 \mathrm{~h}$ before. Figure 9 shows that $\Delta_{24 h} \theta_{\mathrm{se}}$ was greater than zero from the ground to below the $200 \mathrm{hPa}$ ULJ during 0000 UTC 27 to 0600 UTC 27 October 2014, with its center located around $500 \mathrm{hPa}$ at more than $10^{\circ} \mathrm{C}$. This period was the concentrated time of strong precipitation and lightning occurrence (Figure 1). At 1200 UTC 


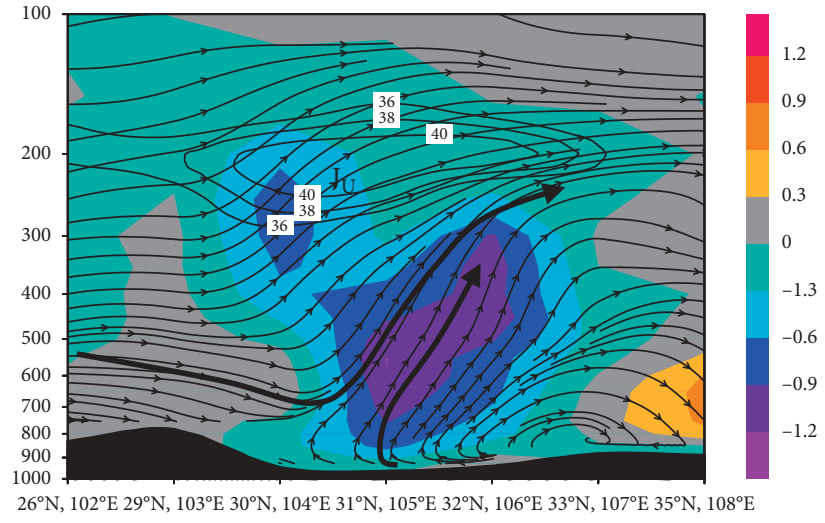

(a)

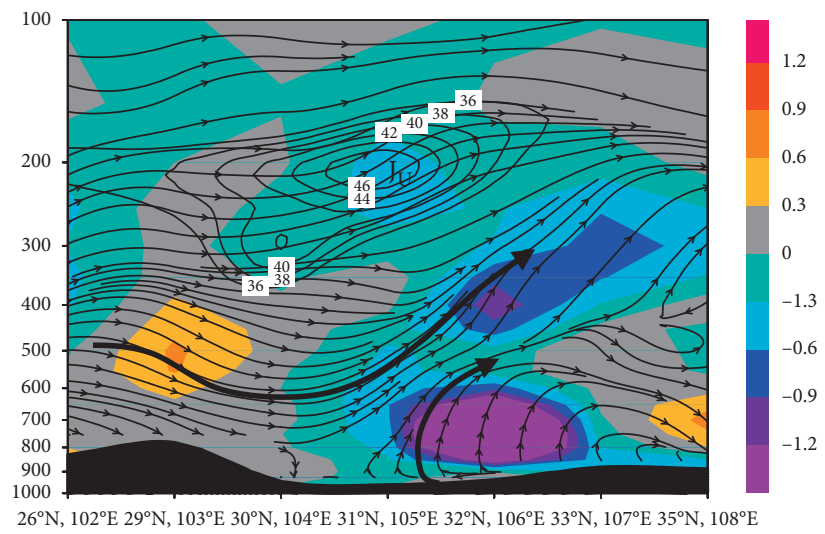

(c)

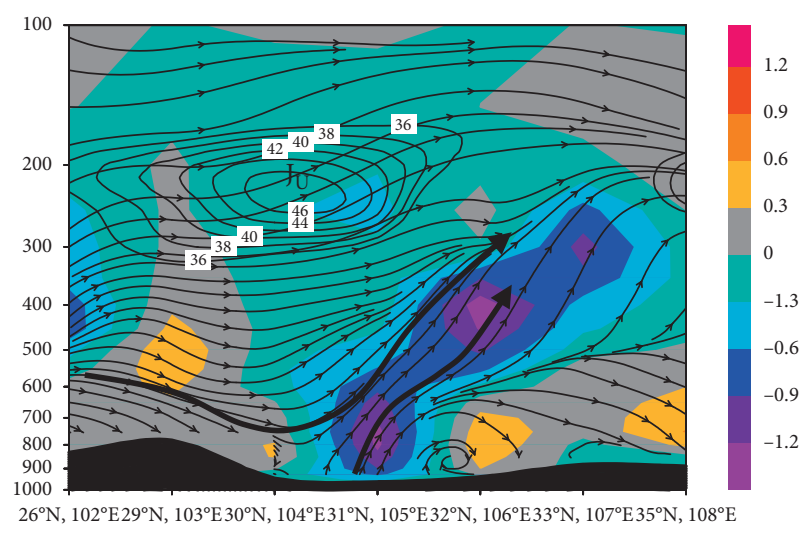

(b)

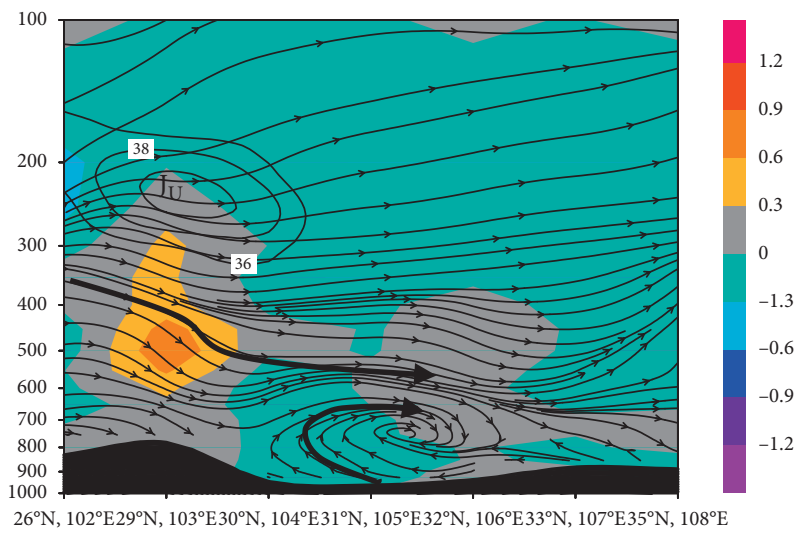

(d)

FiguRE 7: Cross section of vertical circulation along line A-A' in Figure 6(a), in which the solid contours are the horizontal wind speed ("JU" is the ULJ axis), the shaded area is the vertical velocity $\left(10^{-2} \mathrm{~Pa} \cdot \mathrm{s}^{-1}\right)$, the stream line is the synthetic circulation of both horizontal wind $\left(\mathrm{m} \cdot \mathrm{s}^{-1}\right)$ and vertical velocity $\left(10^{-2} \mathrm{~Pa} \cdot \mathrm{s}^{-1}\right)$, the thick solid lines with arrows are the airflow direction, and the black areas are the terrain, at (a) 0000 UTC 27 October 2014, (b) 0600 UTC 27 October 2014, (c) 1200 UTC 27 October 2014, and (d) 0000 UTC 28 October 2014.

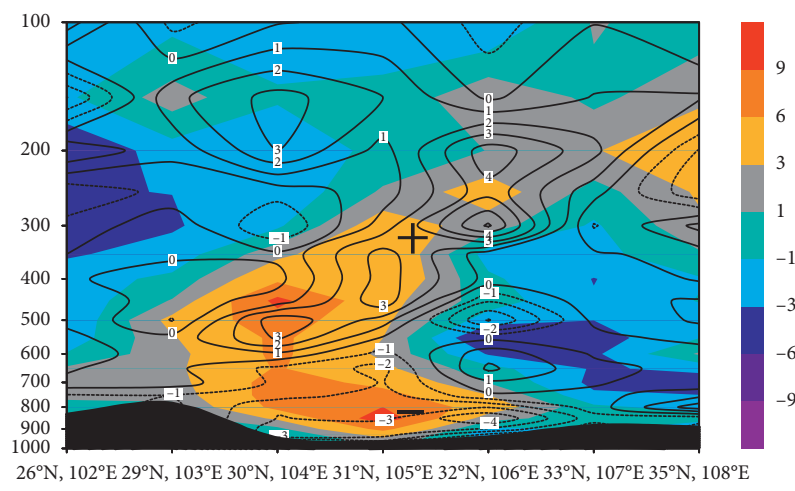

(a)

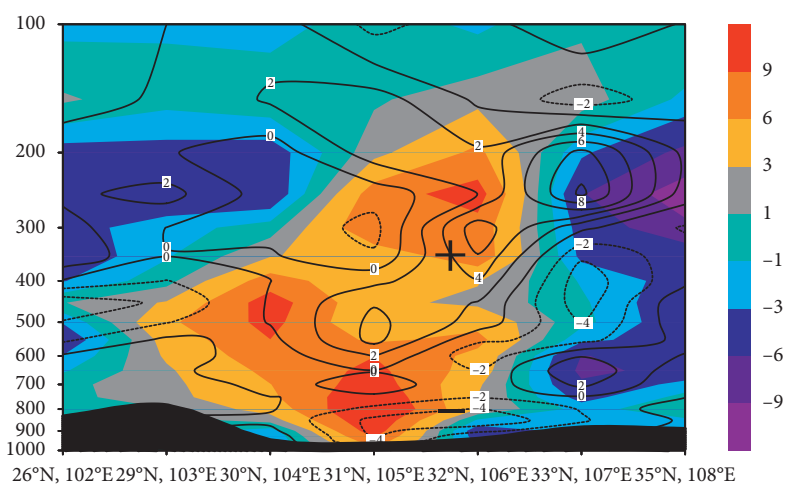

(b)

Figure 8: Continued. 


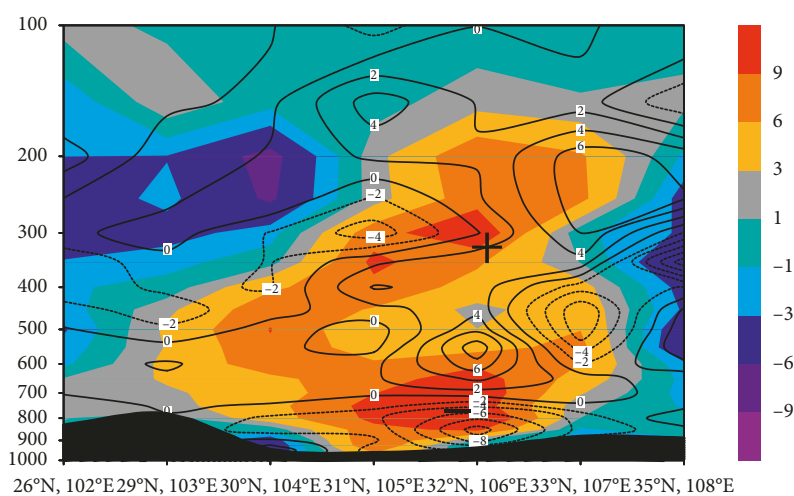

(c)

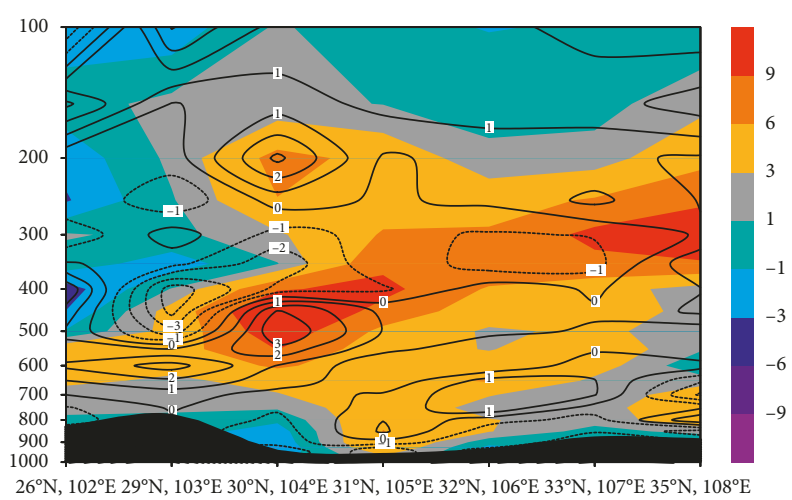

(d)

Figure 8: As in Figure 7 but for the vorticity (shaded, $10^{-5} \mathrm{~s}^{-1}$ ) and divergence (contours, $10^{-5} \mathrm{~s}^{-1}$ ). “-” is convergence; “+” is divergence.

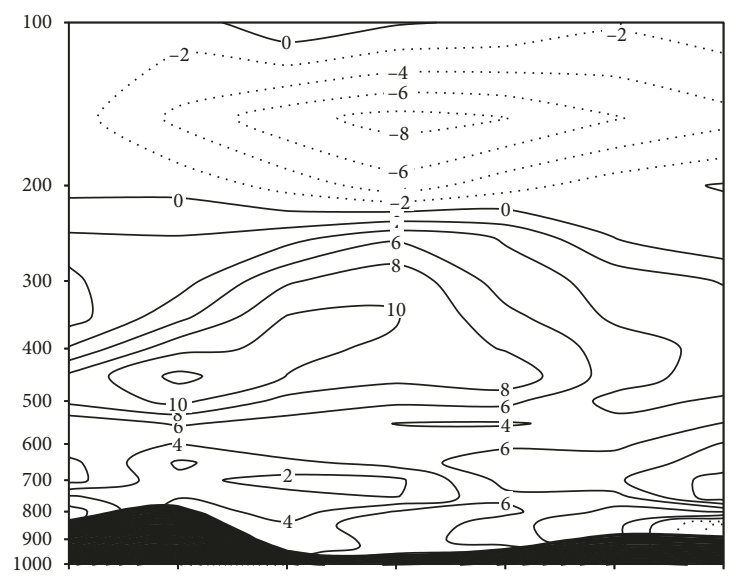

(a)

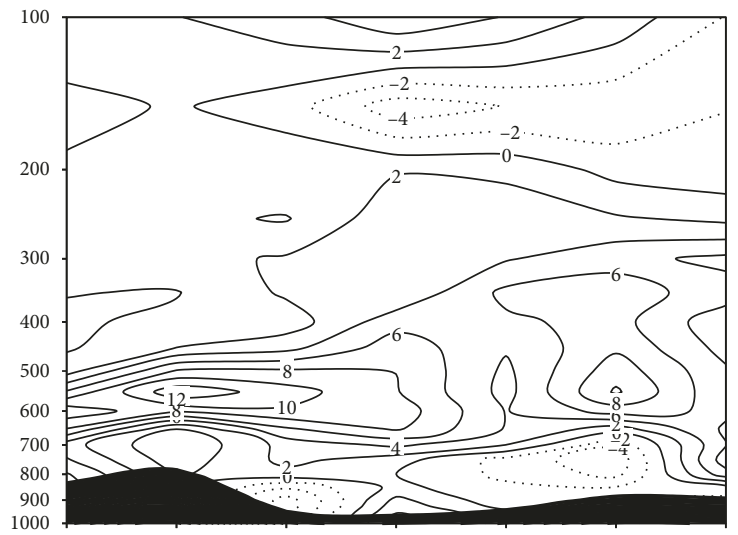

$26^{\circ} \mathrm{N}, 102^{\circ} \mathrm{E} 29^{\circ} \mathrm{N}, 103^{\circ} \mathrm{E} 30^{\circ} \mathrm{N}, 104^{\circ} \mathrm{E} 31^{\circ} \mathrm{N}, 105^{\circ} \mathrm{E} 32^{\circ} \mathrm{N}, 106^{\circ} \mathrm{E} 33^{\circ} \mathrm{N}, 107^{\circ} \mathrm{E} 35^{\circ} \mathrm{N}, 108^{\circ} \mathrm{E}$

(c)

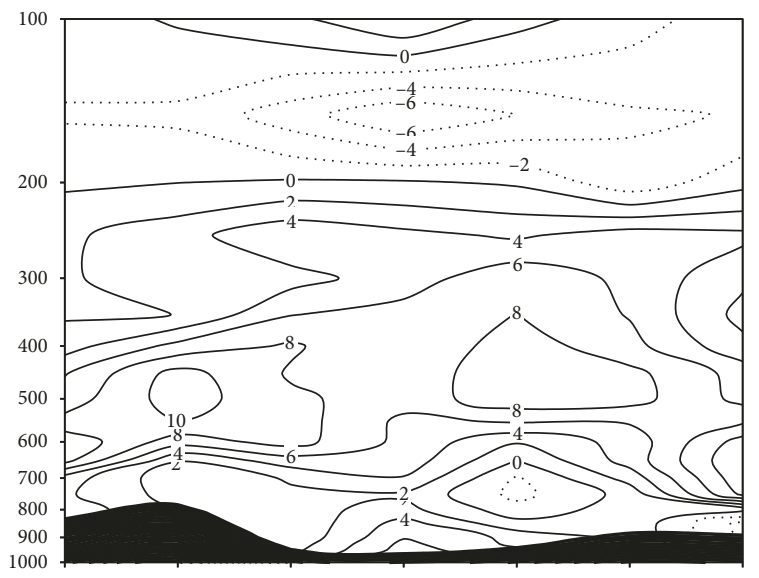

(b)

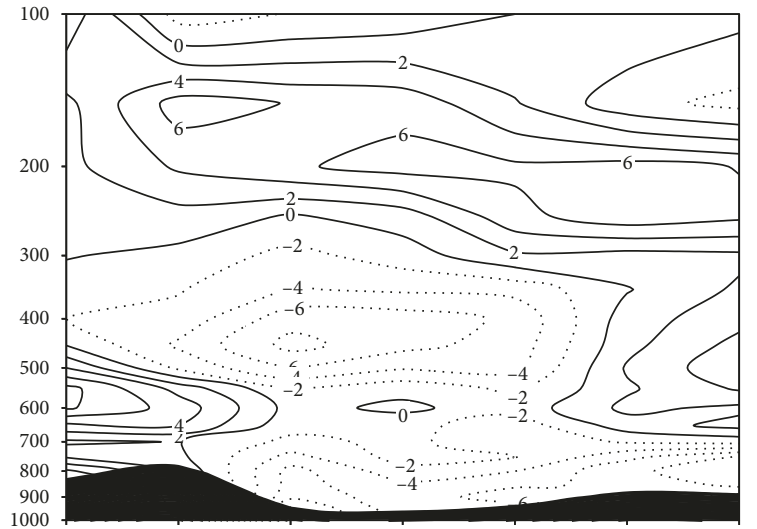

$26^{\circ} \mathrm{N}, 102^{\circ} \mathrm{E} 29^{\circ} \mathrm{N}, 103^{\circ} \mathrm{E} 30^{\circ} \mathrm{N}, 104^{\circ} \mathrm{E} 31^{\circ} \mathrm{N}, 105^{\circ} \mathrm{E} 32^{\circ} \mathrm{N}, 106^{\circ} \mathrm{E} 33^{\circ} \mathrm{N}, 107^{\circ} \mathrm{E} 35^{\circ} \mathrm{N}, 108^{\circ} \mathrm{E}$

(d)

Figure 9: As in Figure 7 but for $\Delta_{24 h} \theta_{\text {se }}\left({ }^{\circ} \mathrm{C}\right)$.

27 October 2014, $\Delta_{24 h} \theta_{\text {se }}$ was less than zero from the ground to $800 \mathrm{hPa}$, but greater than zero from $700 \mathrm{hPa}$ to $200 \mathrm{hPa}$. This showed the PEPT variation was negative in the low-level atmosphere when the low vortex formed. At 0000 UTC 28 October 2014, the negative $\Delta_{24 h} \theta_{\text {se }}$ had expanded to $300 \mathrm{hPa}$ and the central value was $-6^{\circ} \mathrm{C}$. Correspondingly, the precipitation decreased and the number of lightning flashes reduced in this period. The variation in PEPT showed a tendency for the PEPT to increase when the inverted trough evolved toward the SWCV, whereas the PEPT decreased during the SWCV phase and the strong precipitation and lightning activity were in the period of $\Delta_{24 h} \theta_{\text {se }}>0$. 
Based on the characteristics of dynamic and the thermodynamic structure, the vertical upward motion was analyzed. In general, the occurrence of heavy rainfall, even severe convective weather, has a significant relationship with the vertical ascending motion, heavy rainfall areas often located in ascending motion areas. According to quasigeostrophic theory, the vorticity advection and thermodynamic advection are the main factors impacting upon the vertical velocity. When the positive vorticity advection with height increases (or negative vorticity advection with height decreases $),(\partial / \partial p)\left(-\vec{V}_{g} \cdot \nabla \xi_{g}\right)<0$, and in the warm advection area $\left(-\vec{V}_{g} \cdot \nabla \theta_{\text {se }}>0\right) \omega<0$, conditions are conducive to a forced rise; otherwise, there will be a forced sink.

Figure 10 presents cross sections of both vorticity advection (Figures 10(a), 10(c), 10(e), and 10(g)) and PEPT advection (Figures 10(b), 10(d), 10(f), and 10(h)) along the ULJ and LLJ intersection area. Under the $850 \mathrm{hPa}$ inverted trough situation (Figures 10(a) and 10(b)), positive vorticity advection increased with height within $600-200 \mathrm{hPa}$ $\left((\partial / \partial p)\left(-\vec{V}_{g} \cdot \nabla \xi_{g}\right)<0\right)$, with the maximum value of vorticity advection close to the center of the $200 \mathrm{hPa}$ jet. Meanwhile, negative vorticity advection decreased with height within 900-700 hPa and, correspondingly, PEPT advection was positive in the low-level atmosphere and conductive to the formation of upward motion. Up until 0600 UTC 27 October 2014 (Figures 10(c) and 10(d)), lowlevel vorticity advection was negative to the south of $32^{\circ} \mathrm{N}$ and gradually decreased with height but was positive to the north of $32^{\circ} \mathrm{N}$ and increased with height. Also, the vertical distribution of positive (negative) vorticity advection in the lower (upper) atmosphere satisfied $(\partial / \partial p)\left(-\vec{V}_{g} \cdot \nabla \xi_{g}\right)<0$ and still favored the formation of upward motion. For the PEPT advection, there was low-level warm advection north of $32^{\circ} \mathrm{N}$ and cold advection near $31^{\circ} \mathrm{N}$ in the midlevel atmosphere, which was conducive to upward motion in the basin.

When the SWCV formed (Figures 10(e)-10(f)), vorticity advection firstly increased and then decreased with height within $900-500 \mathrm{hPa}$. Also, vorticity advection gradually increased within $500-200 \mathrm{hPa}$, and this distribution was mainly in favor of upward motion in the lower-level south of $32^{\circ} \mathrm{N}$ and upper-level north $32^{\circ} \mathrm{N}$. However, cold PEPT advection in the middle level divided the relationship between the lower-level and upper-level warm advection to a certain extent, and this distribution of middle cold and lower warm was conductive to convective instability and ascending motion in low level. Up until 0000 UTC 28 October 2014, vorticity advection and PEPT advection significantly weakened in the heavy rainstorm area, and the dynamic and thermodynamic variations were no longer in favor of vertical upward motion.

Thus, the vertical distribution of both vorticity advection and PEPT advection played a positive role in promoting the formation of the upward branch of vertical circulation located in the intersection area of the ULJ and LLJ. This is because positive vorticity advection not only caused the positive vorticity to maintain on the north side of the ULJ, but also made the upward motion strengthen near the area of the ULJ and LLJ intersection. On the one hand, the low-level warm advection was beneficial to upward motion, while on the other hand, the configuration with the midlevel cold advection led to the atmosphere over rainstorm area is in a convective unstable state.

In terms of horizontal advection changes, studies have shown that positive vorticity advection in the upper level and warm advection in the lower level have important implications in determining the severe weather process. Miller [43] considered strong positive vorticity advection at $500 \mathrm{hPa}$ to be the most important parameter for severe weather forecasts, and the research of Hales [44] showed that considering the vorticity advection at $250 \mathrm{hPa}$ and horizontal shear can possibly help determine the occurrence of the severe weather area. Maddox and Doswell [45] also pointed out that, when the vorticity field in midlevel troposphere weakens, the warm advection in the low level should be considered as an important factor for forecasting severe weather. Based on the above understanding, we analyzed the $500 \mathrm{hPa}$ vorticity advection and $850 \mathrm{hPa}$ PEPT advection to reflect the advection forcing interaction (Figure 11).

At 0000 UTC 27 October 2014, the $500 \mathrm{hPa}$ vorticity advection was positive over the rainstorm area (value of $3 \times 10^{-9} \mathrm{~s}^{-2}$ ). Then, up until 0600 UTC 27 October 2014, the positive vorticity advection of the heavy rainstorm area weakened, but there were two strong positive vorticity advection zones on the north and south sides of rainfall areas (values of $3 \times 10^{-9} \mathrm{~s}^{-2}$ ). Between 1200 UTC 27 and 0000 UTC 28 October 2014, the positive vorticity advection was in the area from Luzhou to Panzhihua, where it no longer affected the heavy rainstorm zone, and there was a process by which positive vorticity advection weakened in the heavy rain.

From the PEPT advection, during 0000-1200 UTC 27 October 2014, warm advection maintained in the heavy rainstorm. However, up until 0000 UTC 28 October 2014, warm advection was east of $108^{\circ} \mathrm{E}$ and cold advection affected the heavy rain area. Therefore, when the inverted trough evolved toward the SWCV, although the positive vorticity advection of the middle troposphere weakened in the heavy rain area, the warm advection strengthened in the lower troposphere, and this change remained conducive to upward motion. This in turn meant that the convective activity was maintained, because the temperature advection field is a forced item of the quasigeostrophic $\omega$ equation. When positive vorticity advection weakens, its role may become even more important, and when warm advection occurs in the unstable stratification region, it may have a significant impact on the severe weather.

\section{Favorable Conditions for the Occurrence of the Heavy Rainstorm}

6.1. LLJ and Water Vapor Transport. In the strong upward movement, as a wide range of the heavy rainstorm process, the water vapor is an important condition. Since the water vapor transport in the present case was focused in the lower troposphere, we further analyzed the $850 \mathrm{hPa}$ water vapor (Figure 12). During the early period of the inverted trough's 


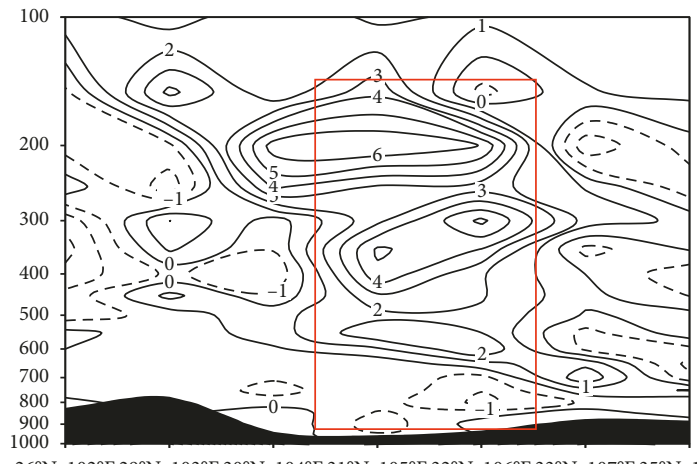

(a)

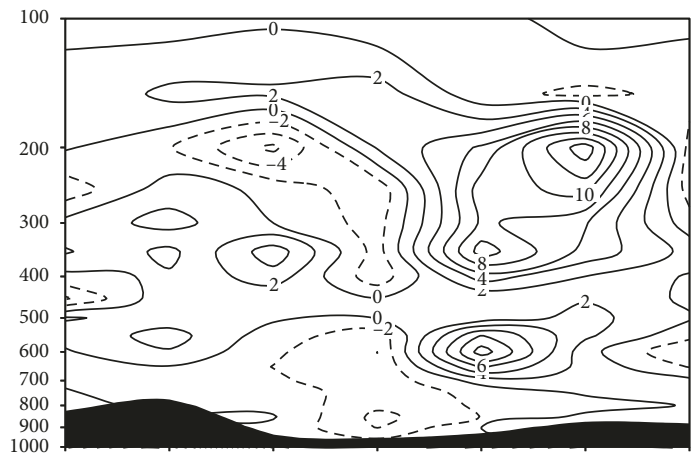

(c)

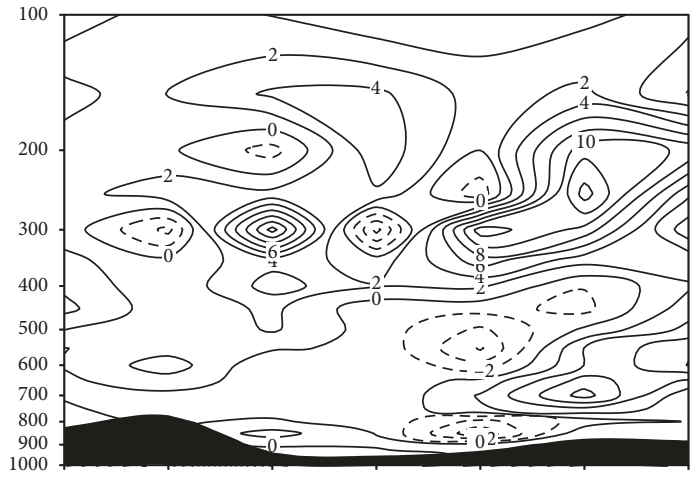

(e)

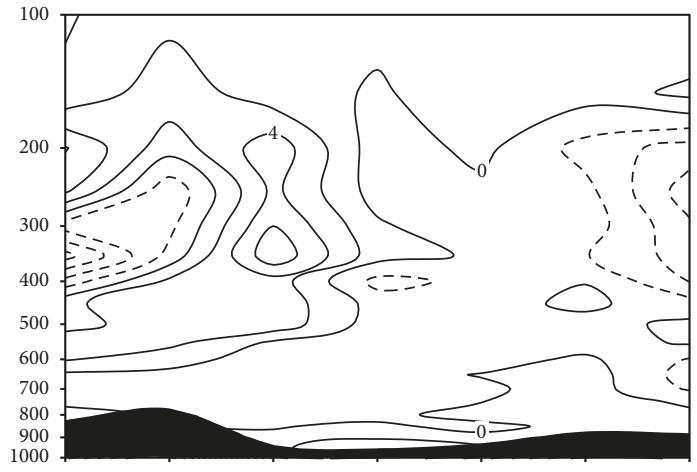

(g)

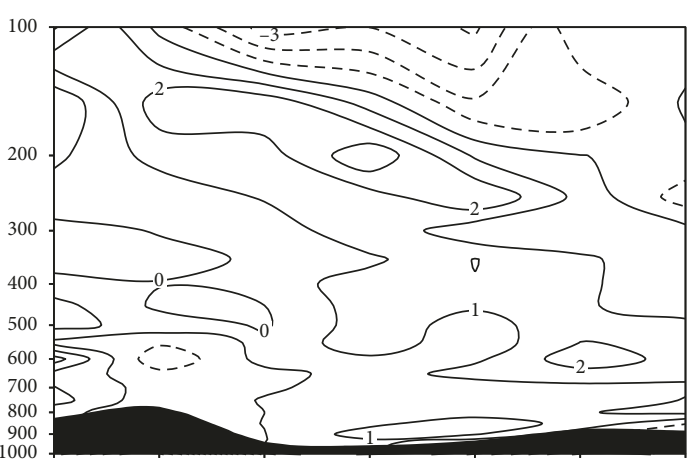

$26^{\circ} \mathrm{N}, 102^{\circ} \mathrm{E} 29^{\circ} \mathrm{N}, 103^{\circ} \mathrm{E} 30^{\circ} \mathrm{N}, 104^{\circ} \mathrm{E} 31^{\circ} \mathrm{N}, 105^{\circ} \mathrm{E} 32^{\circ} \mathrm{N}, 106^{\circ} \mathrm{E} 33^{\circ} \mathrm{N}, 107^{\circ} \mathrm{E} 35^{\circ} \mathrm{N}, 108^{\circ} \mathrm{E}$

(b)

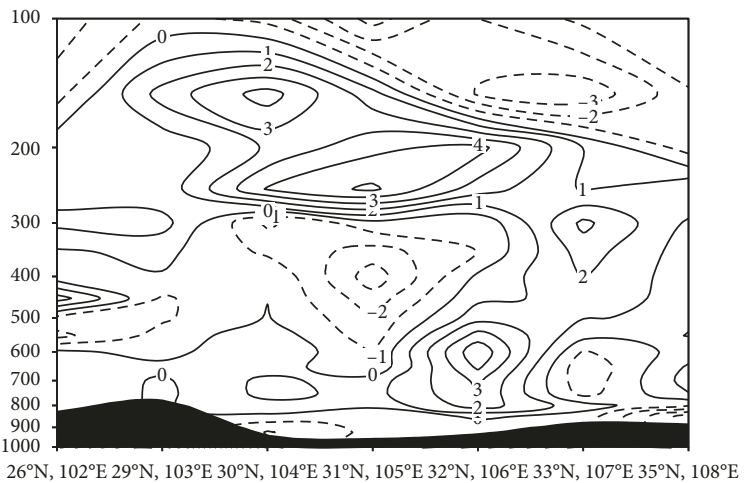

(d)

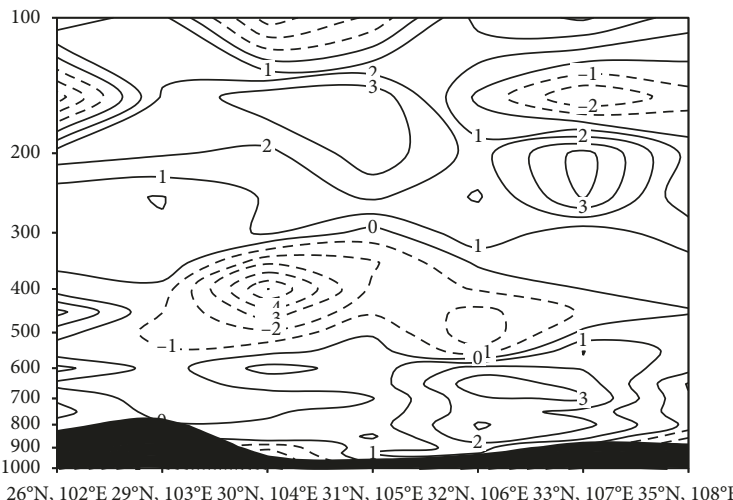

(f)

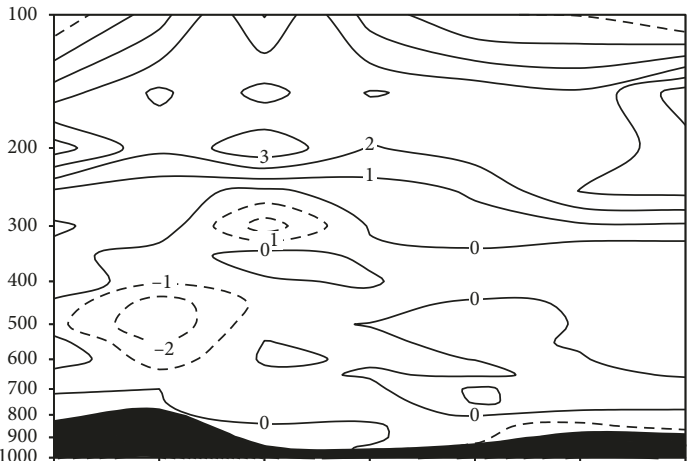

$26^{\circ} \mathrm{N}, 102^{\circ} \mathrm{E} 29^{\circ} \mathrm{N}, 103^{\circ} \mathrm{E} 30^{\circ} \mathrm{N}, 104^{\circ} \mathrm{E} 31^{\circ} \mathrm{N}, 105^{\circ} \mathrm{E} 32^{\circ} \mathrm{N}, 106^{\circ} \mathrm{E} 33^{\circ} \mathrm{N}, 107^{\circ} \mathrm{E} 35^{\circ} \mathrm{N}, 108^{\circ} \mathrm{E}$

(h)

FIgure 10: Cross section of vorticity advection ( $\mathrm{a}, \mathrm{c}, \mathrm{e}, \mathrm{g}$ ) and PEPT advection (b, d, f, h) along line A- $\mathrm{A}^{\prime}$ in Figure 6(a) (black area is the terrain) at (a, b) 0000 UTC 27 October 2014, (c, d) 0600 UTC 27 October 2014, (e, f) 1200 UTC 27 October 2014, and (g, h) 0000 UTC 28 October 2014. In (a) red box area represents rainstorm area. 


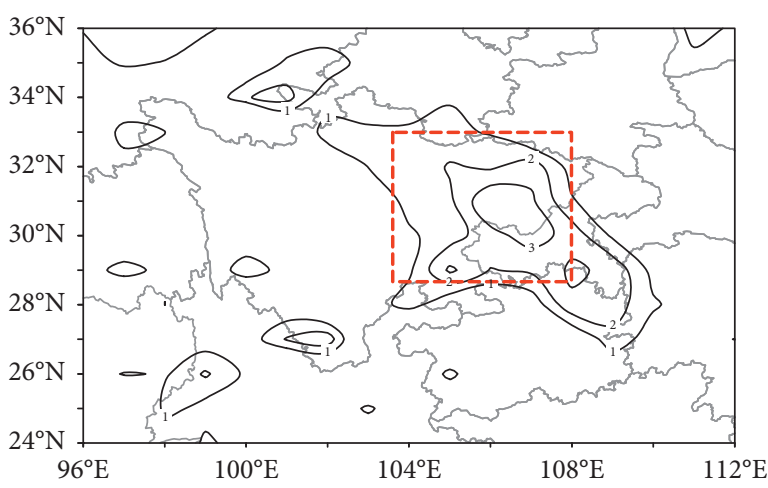

(a)

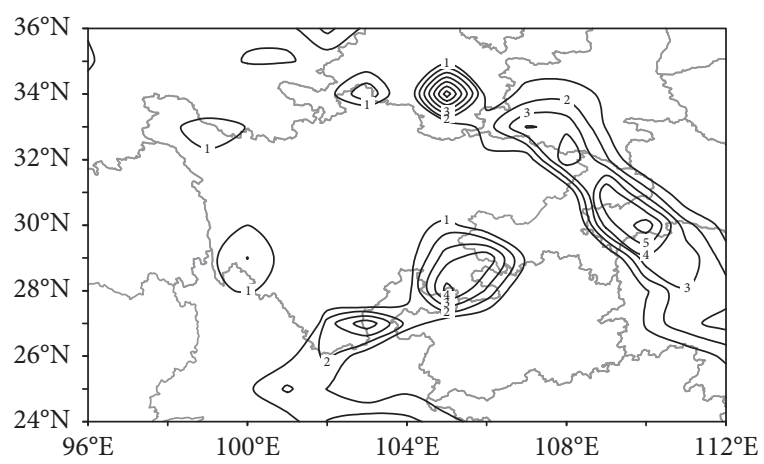

(c)

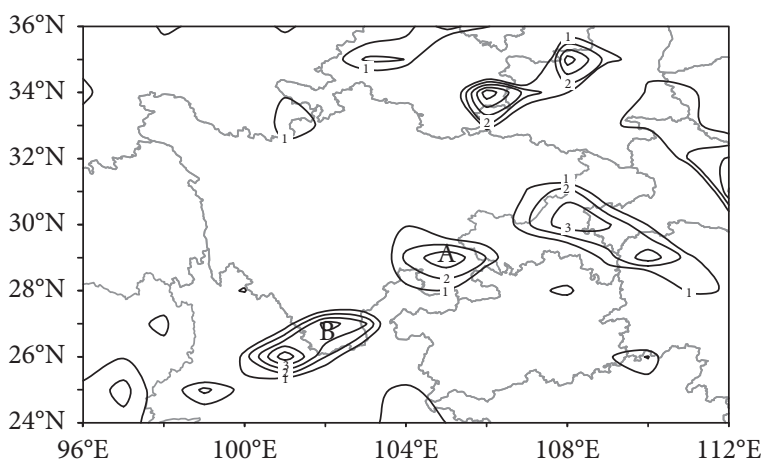

(e)

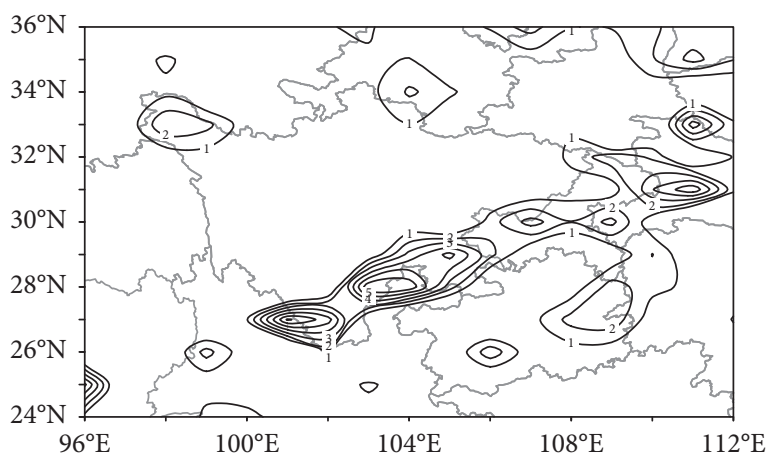

(g)

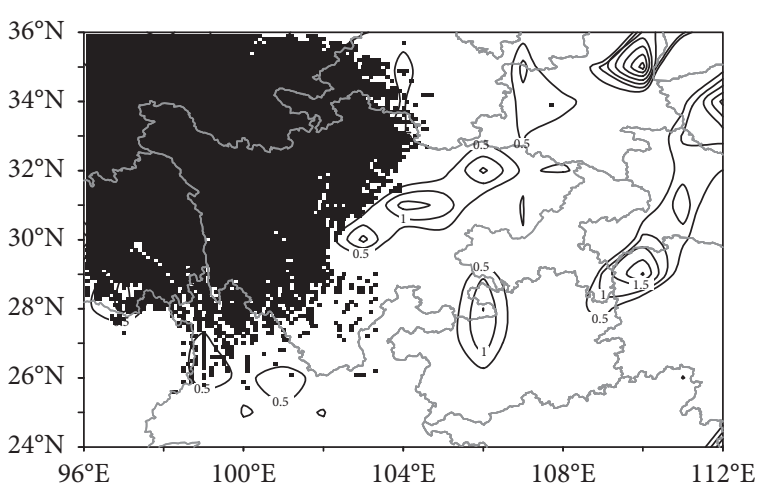

(b)

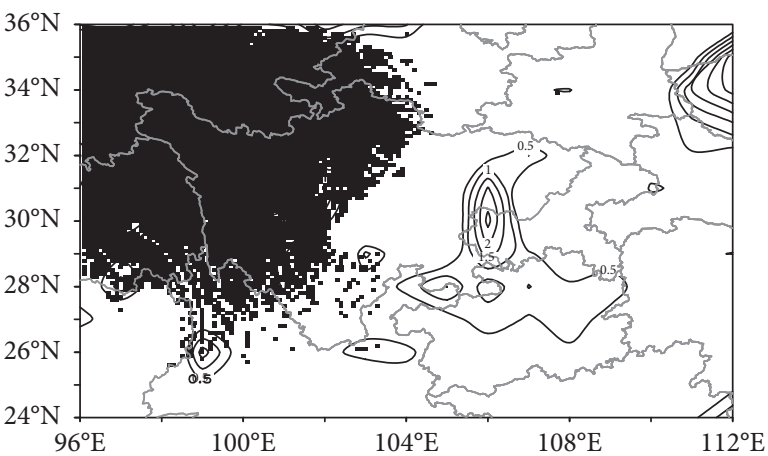

(d)

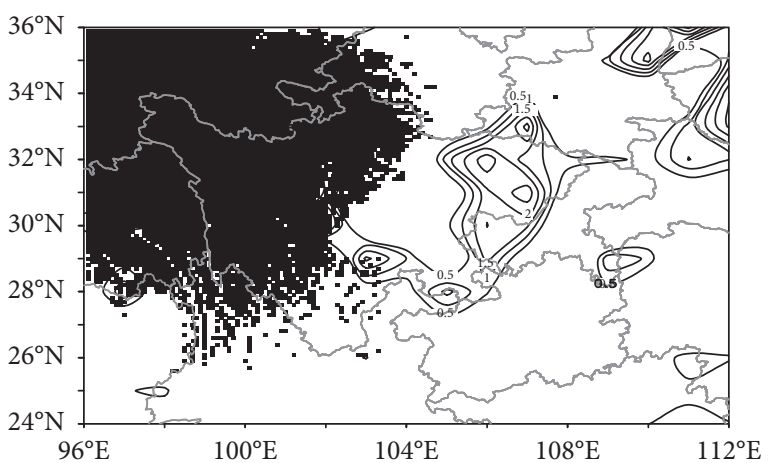

(f)

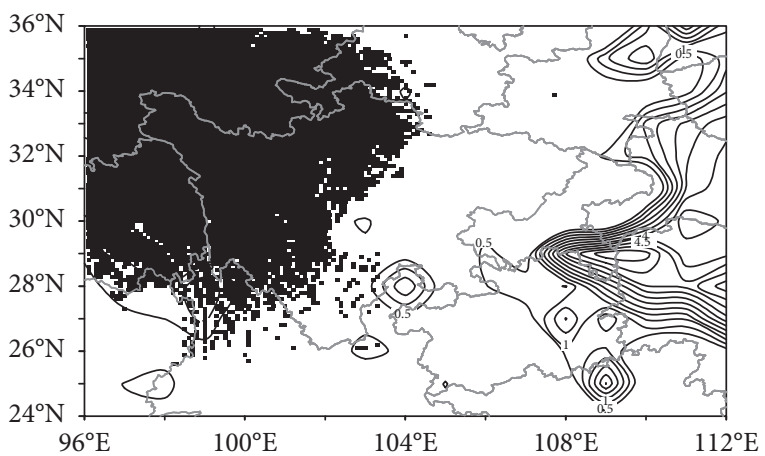

(h)

Figure 11: $500 \mathrm{hPa}$ vorticity advection $(\mathrm{a}, \mathrm{c}, \mathrm{e}, \mathrm{g})\left(10^{-9} \mathrm{~s}^{-2}\right)$ and $850 \mathrm{hPa}$ PEPT advection $(\mathrm{b}, \mathrm{d}, \mathrm{f}, \mathrm{h})\left(10^{-4} \mathrm{~K} \cdot \mathrm{s}^{-1}\right)$ at $(\mathrm{a}, \mathrm{b}) 0000 \mathrm{UTC} 27 \mathrm{October}$ 2014, (c, d) 0600 UTC 27 October 2014, (e, f) 1200 UTC 27 October 2014, and (g, h) 0000 UTC 28 October 2014. In (e), A-Luzhou; B-Panzhihua. 

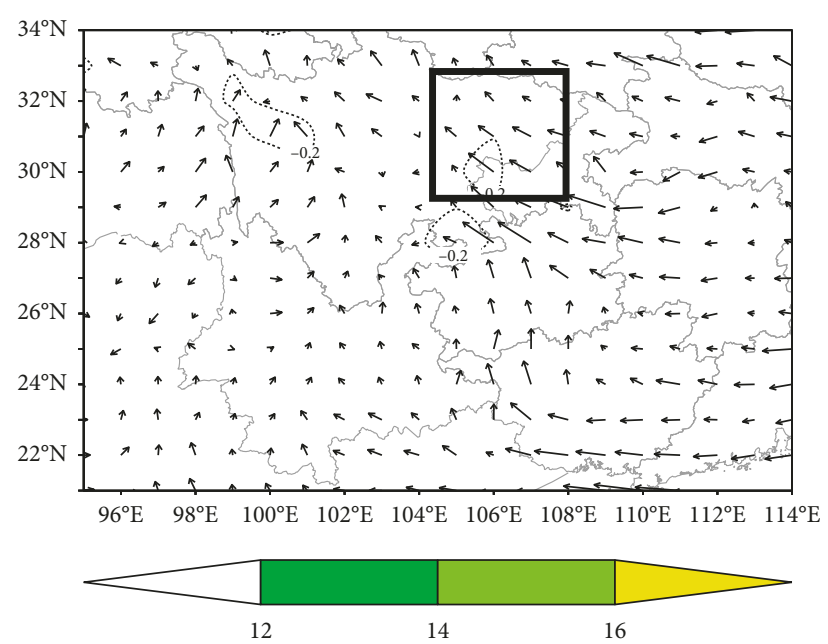

(a)
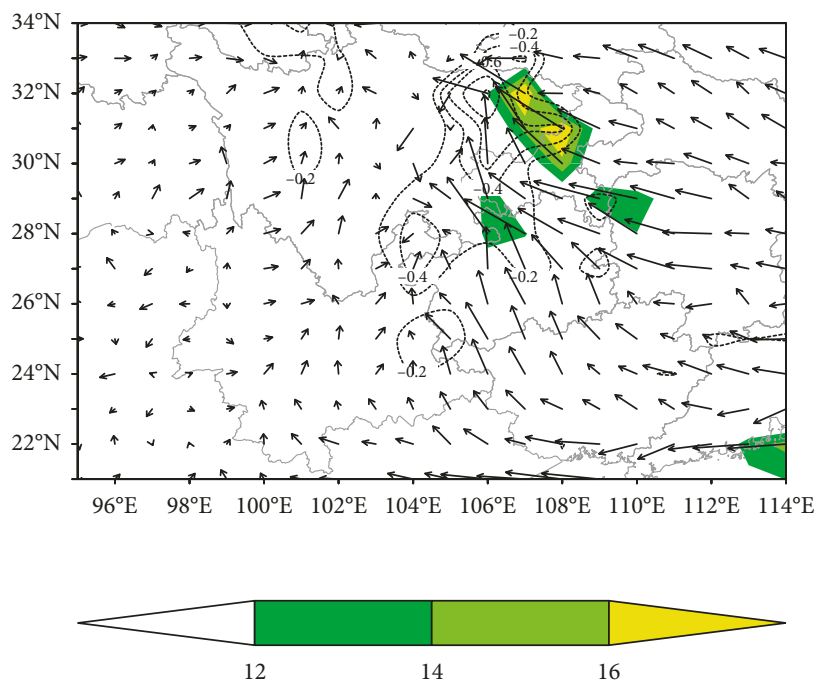

(c)

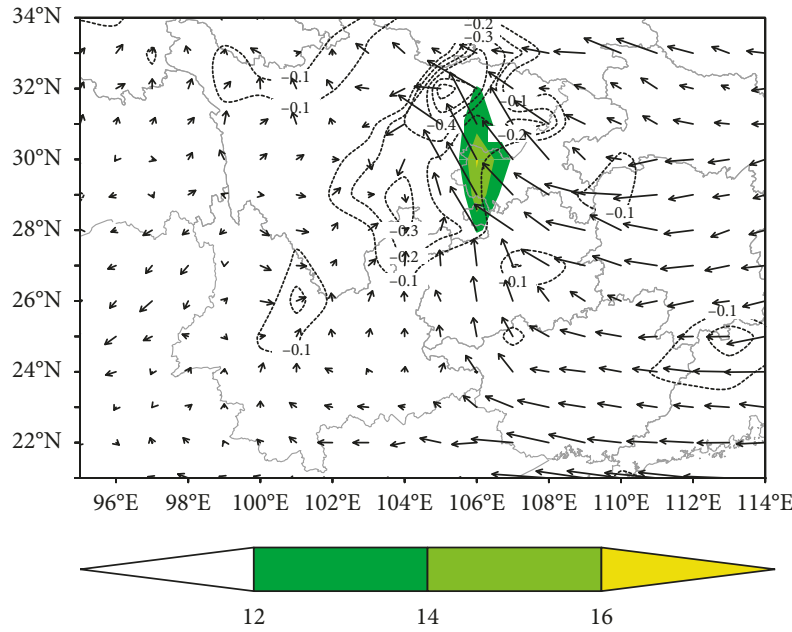

(b)
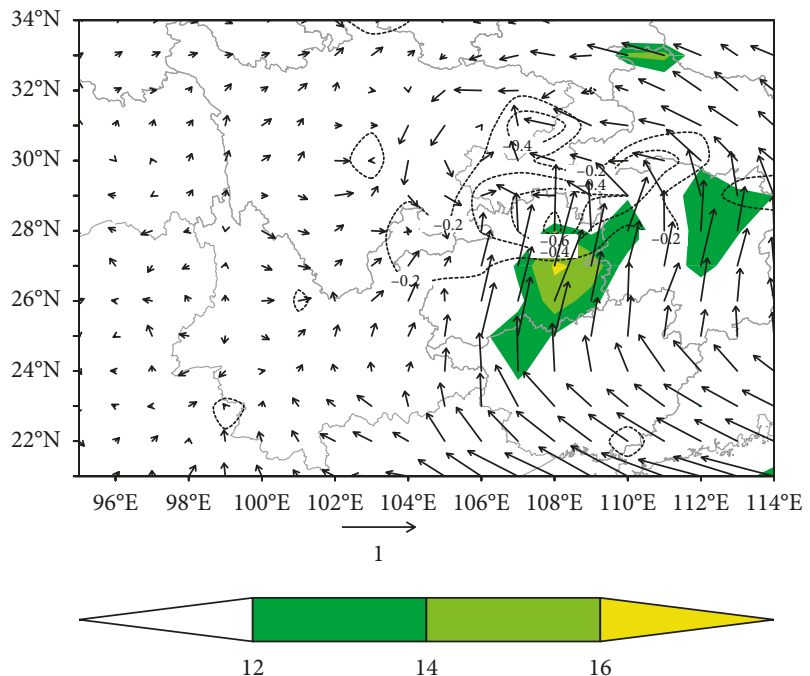

(d)

Figure 12: $850 \mathrm{hPa}$ water vapor flux vector (arrows, $\mathrm{kg} \cdot \mathrm{m}^{-1} \cdot \mathrm{hPa}^{-1} \cdot \mathrm{s}^{-1}$ ) and its divergence (dashed line, $10^{-5} \mathrm{~kg} \cdot \mathrm{m}^{-2} \cdot \mathrm{hPa} \mathrm{a}^{-1} \cdot \mathrm{s}^{-1}$ ) and the area (filled) with wind speed $>12 \mathrm{~m} \cdot \mathrm{s}^{-1}$ at (a) 0000 UTC 27 October 2014, (b) 0600 UTC 27 October 2014, (c) 1200 UTC 27 October 2014, and (d) 0000 UTC 28 October 2014.

formation (Figure 12(a)), the water vapor flux vector was small and convergence was weak. When the inverted trough had formed (Figure 12(b)), the wind field of the eastern basin that was influenced by the large-scale easterly wind enhanced as the LLJ. Also, the water vapor flux vector reached its maximum, and the convergence value reached $-0.5 \times 10^{-5} \mathrm{~kg} \cdot \mathrm{m}^{-2} \cdot \mathrm{hPa}^{-1} \cdot \mathrm{s}^{-1}$ at the position of its turn. When the low vortex had formed (Figure 12(c)), the largevalue area of the vapor flux vector was in the right-hand front of the SWCV and the LLJ continued to be maintained. Water vapor convergence was over the whole basin, but more obvious in the SWCV region, with a value of $-0.6 \times 10^{-5} \mathrm{~kg} \cdot \mathrm{m}^{-2} \cdot \mathrm{hPa}^{-1} \cdot \mathrm{s}^{-1}$. Up until 0000 UTC 28 October 2014, the SWCV remained in the heavy rainstorm area, but due to the jet with water vapor convergence retreating toward the south, this resulted in the low vortex having no rain. Therefore, the LLJ provided water vapor conditions in which the "head" area of the water vapor flux vector or the vector turning point was the large-value zone of moisture convergence, as well as a region of the heavy rainstorm.

6.2. Trigger Conditions and Model of Heavy Rainstorm Occurrence. Under the mutual configuration of the water vapor transport and associated weather systems, the occurrence of the heavy rain also needed a trigger. The PEPT profile lines near the LLJ (Figure 13(a)) showed that PEPT increased from the ground to $850 \mathrm{hPa}$ at 1800 UTC 26 October 2014 and atmospheric state was a stable stratification. However, the PEPT reduced with height from $850 \mathrm{hPa}$ to $550 \mathrm{hPa}$ and atmospheric state was an unstable stratification, which showed that there was stable stratification near the ground in the early stage of the inverted trough's formation.

At 0000 UTC 27 October 2014, the PEPT reduced with height form near the ground to $600 \mathrm{hPa}$ and atmospheric 


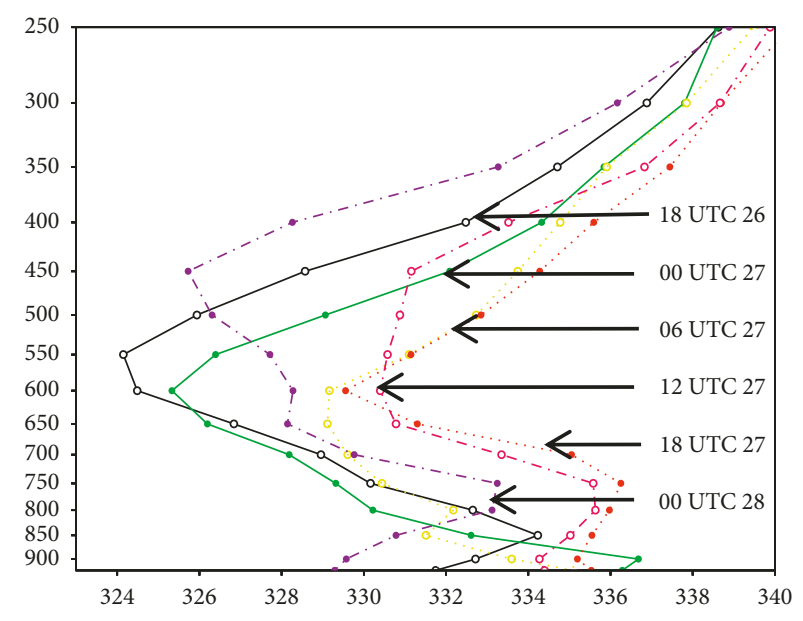

(a)

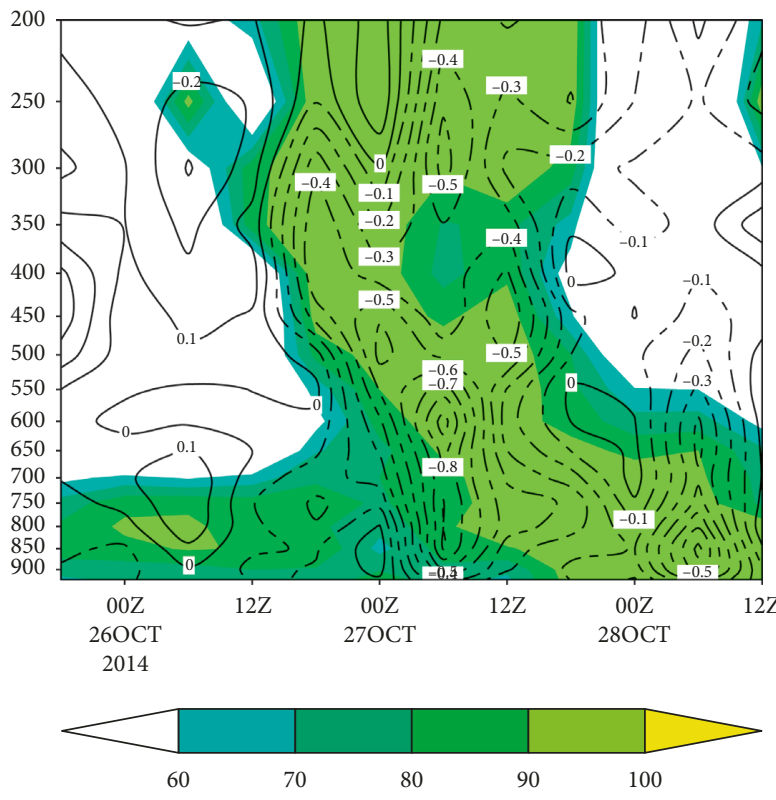

(c)

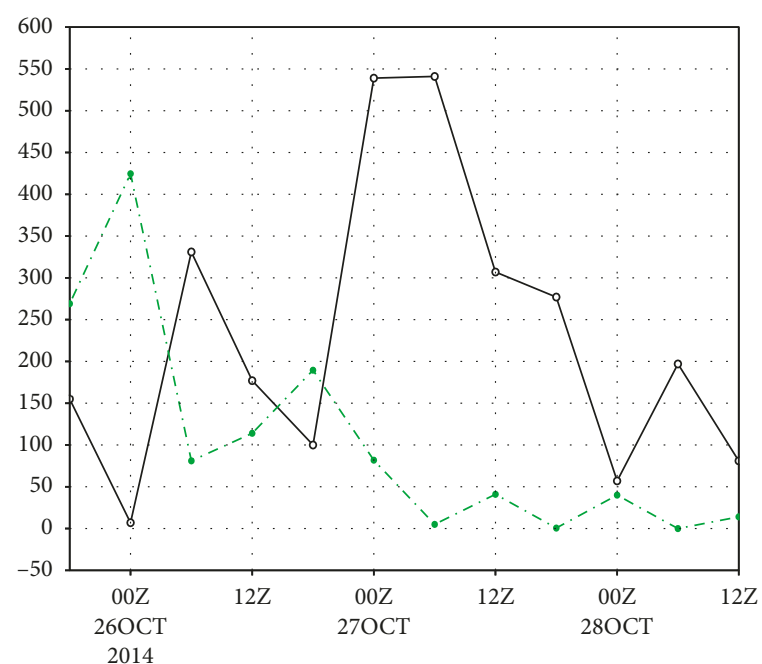

(b)

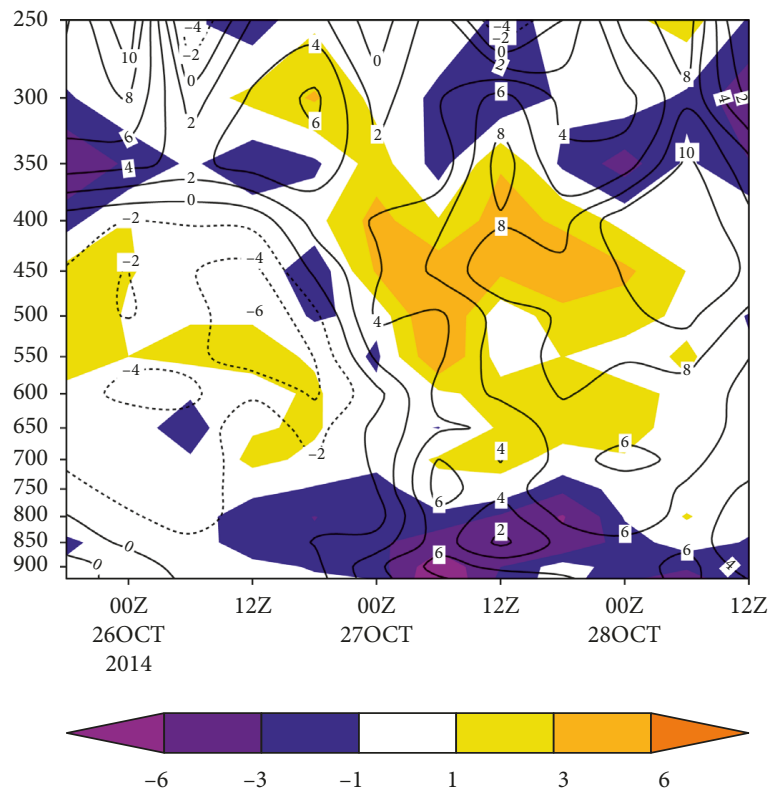

(d)

FIgURE 13: The $\theta_{\text {se }}$ profile line distribution at $\left(106^{\circ} \mathrm{E} 30^{\circ} \mathrm{N}\right)(\mathrm{K})(\mathrm{a})$; the CAPE (solid line, $\left.\mathrm{J} \cdot \mathrm{kg}^{-1}\right)$, and CIN (dashed line, $\left.\mathrm{J} \cdot \mathrm{kg}^{-1}\right)(\mathrm{b})$; the vertical velocity $\left(\mathrm{Pa} \cdot \mathrm{s}^{-1}\right)(\mathrm{c})$; and the vorticity (solid line) and divergence (shading) $\left(10^{-5} \mathrm{~s}^{-1}\right)(\mathrm{d})$.

state was in an unstable stratification. Notably, the atmosphere became unstable from the ground to $850 \mathrm{hPa}$ when the inverted trough formed. At 0600 UTC 27 October 2014, the PEPT profile line was consistent with that at 0000 UTC 27 October 2014, and this period was the time during which the heavy rainstorm occurred and there was frequent lightning activity. At 1200 UTC 27 October 2014, the atmospheric stratification was in a stable state from the land surface to $750 \mathrm{hPa}$ but was unstable from $750 \mathrm{hPa}$ to $500 \mathrm{hPa}$. This indicated that the atmosphere was unstable at the top of the SWCV, but stable at its foot. At 1800 UTC 27 October 2014, the PEPT profile line was consistent with that at 1200 UTC 27 October 2014. Then, at 0000 UTC 28 October 2014, the PEPT profile line became more stable from the surface to $800 \mathrm{hPa}$.
As seen from the convective available potential energy (CAPE) and convective inhibition (CIN) evolution (Figure 13(b)), there was a certain CIN in the early stage of the inverted trough's formation; plus, the CAPE was small. When the inverted trough formed, the CIN decreased and the CAPE increased rapidly. These changes not only provided the favorable conditions for convective development, but also, with the occurrence of strong precipitation and the effect of thunderstorms on the instability of unstable energy dissipation, the CAPE gradually decreased and approached zero at 0000 UTC 28 October 2014. Therefore, the strong precipitation process occurred in the period of larger CAPE and smaller CIN, demonstrating the importance of unstable energy conversion for the heavy rainstorm. 
Because the CAPE conversion had an effect on upward motion, theoretically, the maximum vertical speed and CAPE have the relationship [46] $\omega_{\max } \approx(2 \mathrm{CAPE})^{1 / 2}$, meaning when CAPE is larger, it is more favorable to upward motion and, in particular, it can cause a heavy rainstorm in an area of adequate water vapor.

Before 0000 UTC 27 October 2014 (Figure 13(c)), the velocity of vertical ascent was low and focused in the lowlevel atmosphere. During 0000 UTC 27 to 1800 UTC 27 October 2014, the vertical velocity strengthened and reached its maximum at 0600 UTC 27 October 2014. Correspondingly, the relative humidity became more intense, with the greater than $90 \%$ scope reaching $200 \mathrm{hPa}$.

From the vorticity and divergence distribution (Figure 13(d)), it is clear that these too increased during the period of strengthening precipitation. The increasing positive vorticity was focused in the middle troposphere (400-600 hPa) and the convergence enhanced in the lower levels (900-750 hPa), forming a structure composed of intense positive vorticity and convergence in the troposphere. In the lower level, the maximum divergence value was $-6 \times 10^{-5} \mathrm{~s}^{-1}$ and the maximum positive vorticity was $6 \times 10^{-5} \mathrm{~s}^{-1}$, whereas in the upper level, the divergence value was $3 \times 10^{-5} \mathrm{~s}^{-1}$ and the positive vorticity was $10 \times 10^{-5} \mathrm{~s}^{-1}$. These physical conditions played a crucial role in the heavy rainstorm process.

We further analyzed the $850 \mathrm{hPa}$ wet zone and atmospheric column precipitable water (Figure 14). At 1800 UTC 26 October 2014, with the heavy rain approaching (not shown), the heavy rainstorm area in the basin was a wet zone (temperature dew-point difference less than $5^{\circ} \mathrm{C}$ ) and the precipitable water value reached $30-35 \mathrm{~mm}$. However, the temperature dew-point difference was more than $10^{\circ} \mathrm{C}$ in the northeastern part of the basin. The change in the horizontal gradient of humidity was large, forming a dew-point front (it is an air mass boundary marked by a strong moisture gradient, also called dry line) $[47,48]$, which was one of the trigger systems in this heavy rainstorm. Up until 0000 UTC 27 October 2014, the dew-point front remained at the northeastern edge of the basin, and there was a similar "tongue" of high precipitable water (maximum of $50 \mathrm{~mm}$ ) forming from low latitudes to the wet area of the basin (abbreviation: wet tongue). During 0600-1200 UTC 27 October 2014, the dew-point front and wet tongue remained in the basin. At 0000 UTC 28 October 2014, the basin was still a wet area but the dew-point front had disappeared; that is, the dew-point front and the greater than $40 \mathrm{~mm}$ precipitable water zone had maintained during the approach and occurrence of the heavy rainstorm. Influenced by the dew-point front and interaction of the ULJ and LLJ, an upward motion was built in the wet zone and descent in a dry area, forming a vertical circulation system (Figure 7). Therefore, the dew-point front led to the increase in the humidity gradient and stimulated upward motion near the LLJ in the wet zone. Moreover, the MCS activities were in part on the wet side of the dew-point front.

In short, from the above analysis, a schematic illustration of the occurrence of this heavy rainstorm can be summarized as follows (Figure 15):
(1) Weather system configuration: The $200 \mathrm{hPa}$ ULJ $(\geq 40 \mathrm{~m} / \mathrm{s})$ and $850 \mathrm{hPa}$ LLJ $(12-16 \mathrm{~m} / \mathrm{s})$ intersected in the heavy rainstorm area of $\mathrm{SB}$, and there was inclined upward motion in the direction of the two branch jets' intersection. A weak vertical circulation system was near the LLJ, with the ascending branch on the wet-zone side of the $850 \mathrm{hPa}$ dew-point front and the descending branch in the dry zone of the northern side of the dew-point front.

(2) Physical impact factors of vertical upward movement: the diagnostic analysis of $\omega$ equation shows the vertical upward movement was caused by both the vorticity advection and low-level warm advection superimposed upon the ascending branch of the vertical circulation in the wet zone. The convergence of the water vapor flux increased and unstable stratification strengthened.

(3) The CPAE value increased and the CIN value decreased, and there was a weak vertical circulation system in the lower levels produced by the combined action of the dew-point front and lower-layer wet zone.

(4) There was MCS activity in the heavy rainstorm area featuring $\mathrm{Tb}$ values of less than $-40^{\circ} \mathrm{C}$ and a radar echo of $35-50 \mathrm{dBZ}$ in the cold-cloud area. Also, a smaller-scale vortex, shear, and low-level jets were observed in the MCS.

\section{Conclusion}

Through analysis of a heavy rain event that occurred on 27 October 2014 in the SB, China, the following conclusions regarding this abnormally heavy rainstorm can be drawn:

(1) When the $850 \mathrm{hPa}$ basin trough evolved toward the SWCV, strong upward motion formed via the interaction between the ULJ and LLJ, and this was the main reason for the occurrence of the heavy rain. Influenced by the large-scale circulation of the subtropical high and westerly trough, two cloud bands formed in the northeastern part of the Tibetan Plateau and the south side of the Tibetan Plateau, respectively. Owing to the trough, LLJ, and low vortex affecting the heavy rainstorm area, an MCS with $\mathrm{Tb}$ values of $40-50^{\circ} \mathrm{C}$ formed, and it was a direct system. The MCS strengthened under the situation of the inverted trough, gradually weakened as the inverted trough evolved toward the low vortex, and lasted about $8 \mathrm{~h}$. During the period of SWCV circulation in the basin, the MCS gradually moved eastward out of the basin and formed the phenomenon known as "vortex but no cloud." In the MCS, there were also smaller-scale systems like small-scale vortices, shears, and jets, which possessed $\beta$-middle and $\gamma$-middle characteristics. The localized rainfall caused by these systems was very strong and usually was a strong precipitation center. 


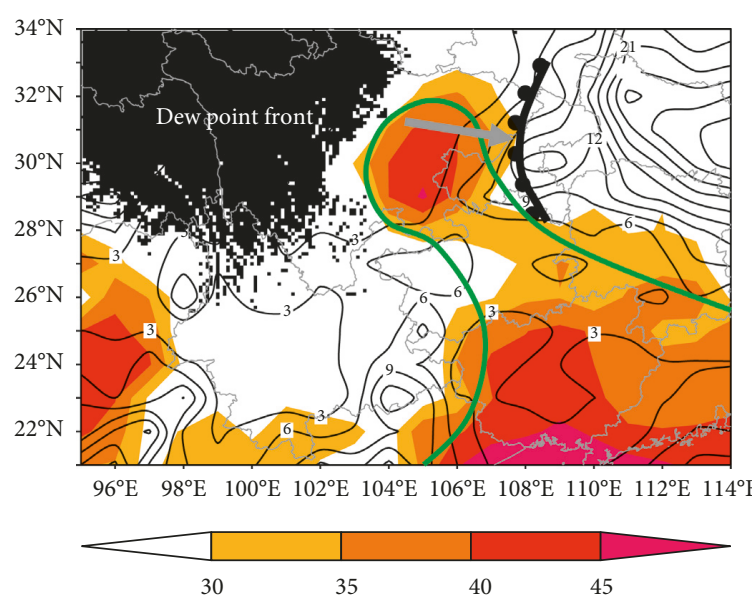

(a)

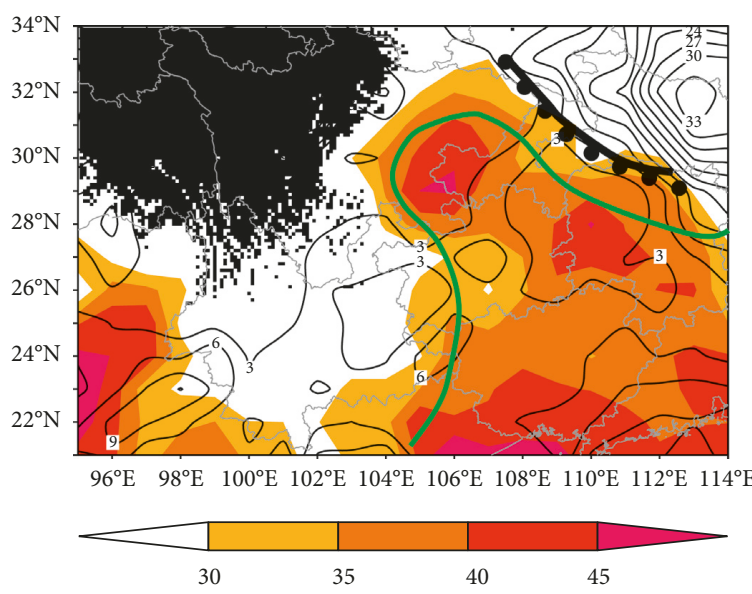

(c)

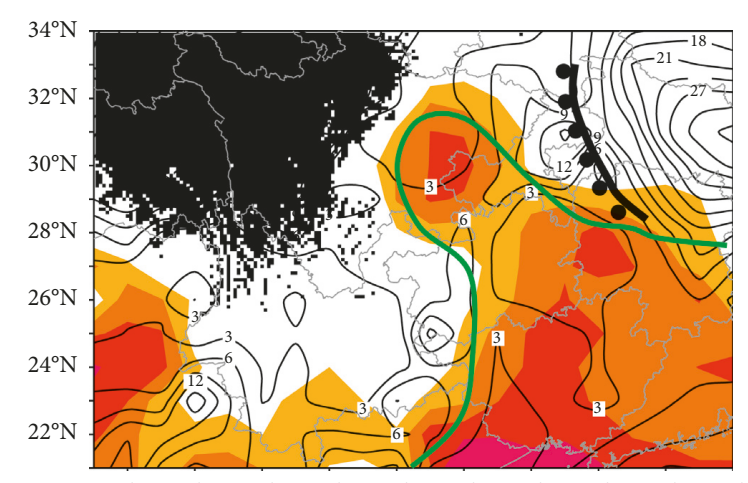

$96^{\circ} \mathrm{E} \quad 98^{\circ} \mathrm{E} 100^{\circ} \mathrm{E} 102^{\circ} \mathrm{E} 104^{\circ} \mathrm{E} 106^{\circ} \mathrm{E} 108^{\circ} \mathrm{E} 110^{\circ} \mathrm{E} 112^{\circ} \mathrm{E} 114^{\circ} \mathrm{E}$

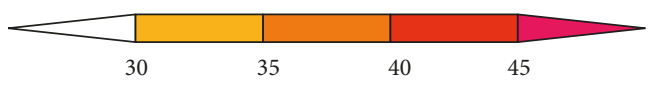

(b)
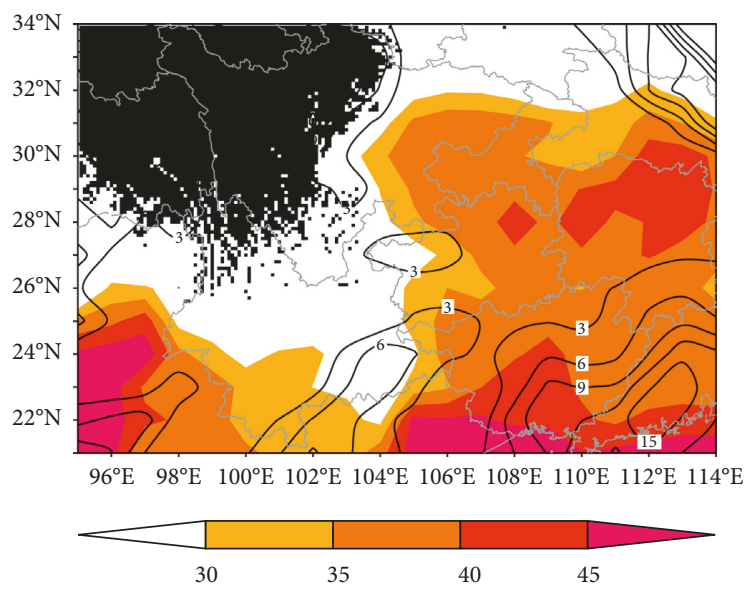

(d)

FIGURE 14: $850 \mathrm{hPa}$ temperature dew-point difference (contours, ${ }^{\circ} \mathrm{C}$ ) and the precipitable water (colored area, $\mathrm{mm}$ ) at (a) $0000 \mathrm{UTC} 27$ October 2014, (b) 0600 UTC 27 October 2014, (c) 1200 UTC 27 October 2014, and (d) 0000 UTC 28 October 2014.

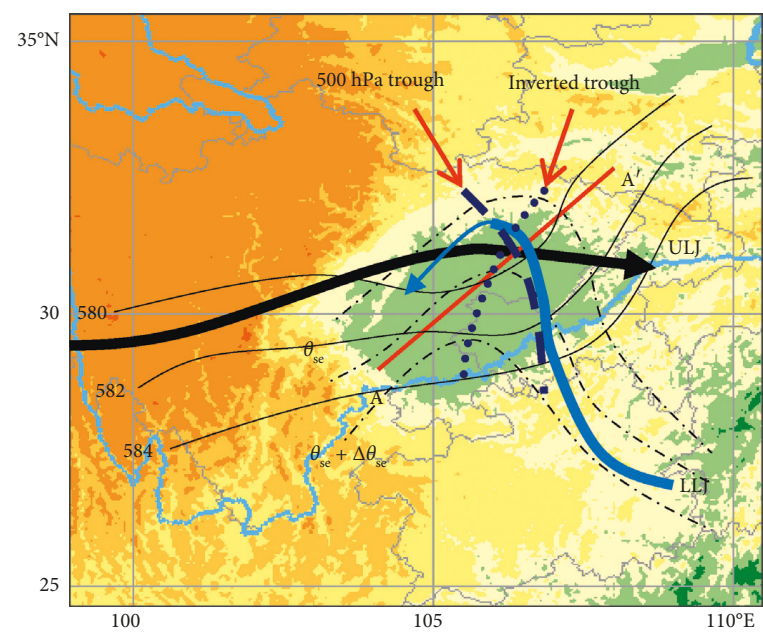

(a)

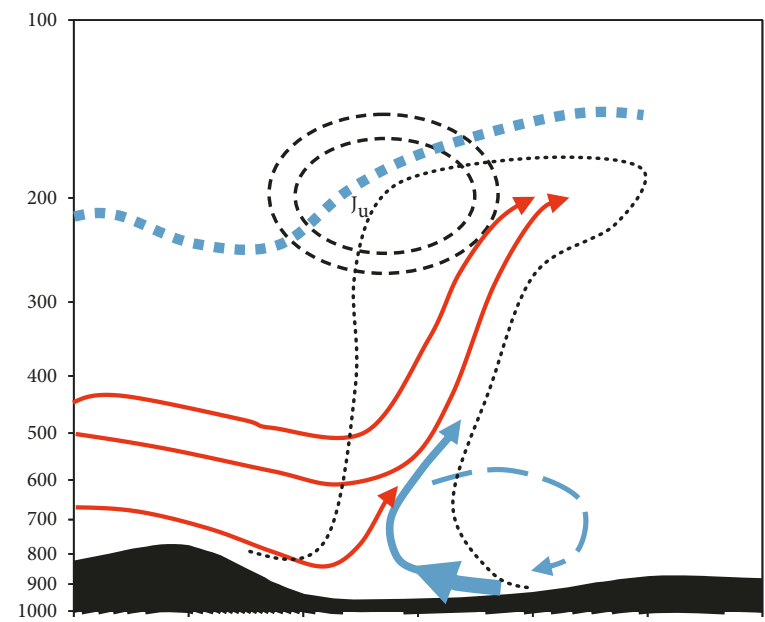

$26^{\circ} \mathrm{N}, 102^{\circ} \mathrm{E} 29^{\circ} \mathrm{N}, 103^{\circ} \mathrm{E} \quad 30^{\circ} \mathrm{N}, 104^{\circ} \mathrm{E} 31^{\circ} \mathrm{N}, 105^{\circ} \mathrm{E} \quad 32^{\circ} \mathrm{N}, 106^{\circ} \mathrm{E} 33^{\circ} \mathrm{N}, 107^{\circ} \mathrm{E} \quad 35^{\circ} \mathrm{N}, 108^{\circ} \mathrm{E}$

(b)

FIGURE 15: A schematic illustration of weather system configuration (a) and schematic diagram of the vertical circulation (b) in this abnormal heavy rainfall. In (a), LLJ-low-level jet; ULJ-upper-level jet; the solid contours-500 hPa geopotential height (units: gpdam); the broken contours-the contours of pseudoequivalent potential temperature $\left(\theta_{\text {se }}\right.$, units: $\left.{ }^{\circ} \mathrm{C}\right)$. In $(\mathrm{b}), \mathrm{J}_{\mathrm{u}}$ - the ULJ axis. 
Therefore, large-scale weather systems affected the range of the precipitation's configuration, while the smaller-scale systems affected the intensity of rainfall.

(2) The heavy rainfall strengthened in the inverted trough and weakened during the low vortex. The cause of the strengthening of precipitation was that there was inclined upward motion in the intersection of the ULJ and LLJ. On the one hand, the upward motion was related to the vorticity advection with height and low-level warm advection, whilst on the other hand, the dew-point front near the LLJ was also beneficial to the ascending branch of the vertical circulation. Meanwhile, the LLJ "head" was a largevalue area of water vapor convergence and provided abundant moisture for heavy rainfall. The reason for the weakening of the precipitation was that the ULJ was interrupt in the SB. The upper airflow presented downdrafts and its superposition with the ascending branch of low-level vertical circulation, this airflow structure inhibited the development of strong upward motion. In addition, the LLJ of the basin retreated southward and the dew-point front disappeared, meaning the moisture convergence weakened and the occurrence of heavy rainfall was no longer supported.

(3) The trigger conditions of the heavy rain were that when the heavy rain began, the CAPE increased and the CIN decreased rapidly, which provided unstable energy for the occurrence of heavy rain. When the heavy rain weakened toward the end of the event, the CAPE and CIN reduced. During this process, the side of the dew-point front in the wet area appeared as a wet tongue and the atmospheric stratification was in an unstable state. Under the influence of the dew-point front, the wet layer thickened and contributed to the development of the MCS, and then the heavy rainstorm and thunderstorm occurred. Based on the above analysis, a schematic illustration of the occurrence of this heavy rainstorm was produced.

\section{Data Availability}

All the data used in this study are as follows: (1) sounding observational data at 0000 UTC and 1200 UTC, and land surface precipitation data are from observational systems of China Meteorological Administration. The datasets generated during and/or analyzed during the current study are available from the corresponding author on reasonable request. (2) Global final (FNL) analysis data from the National Centers for Environmental Prediction (NCEP), with a spatial resolution of $1^{\circ} \times 1^{\circ}$ and temporal resolution of $6 \mathrm{~h}$ (hereafter referred to as NCEP_fnl data); the data can be freely downloaded from the link http://rda.ucar.edu/. (3) Brightness temperature (Tb) data from the FY2D geostationary weather satellite provided by the National Satellite Meteorological Center (NSMC), China Meteorological Administration (http://www.nsmc.org. cn/NSMC/Home/Index.html).

\section{Conflicts of Interest}

The authors declare that there are no conflicts of interest regarding the publication of this paper.

\section{Acknowledgments}

This work was jointly sponsored by a special project of Strategic Science and Technology (Class A) in Chinese Academy of Sciences (XDA23090103), a project of the State Key Laboratory of Severe Weather (LASW2012-A03), a key project of the Applied Basic Research Plan of Sichuan Province of China (no. 2016JY0046), a key project of the National Natural Science Foundation of China (no. 91337215), and National Natural Science Foundation of China (no. 91544109). The authors are grateful to the strong convection forecasting team of Sichuan Meteorological Administration for the assistance they provided.

\section{References}

[1] Y. Chen and P. Zhai, "Persistent extreme precipitation events in China during 1951-2010," Climate Research, vol. 57, no. 2, pp. 143-155, 2013.

[2] Q. Dong, X. Chen, and T. Chen, "Characteristics and changes of extreme precipitation in the Yellow-Huaihe and YangtzeHuaihe rivers basins, China," Journal of Climate, vol. 24, no. 14, pp. 3781-3795, 2011.

[3] S.-Y. Tao and Y.-H. Ding, "Observational evidence of the influence of the Qinghai-Xizang (Tibet) Plateau on the occurrence of heavy rain and severe convective storms in China," Bulletin of the American Meteorological Society, vol. 62, no. 1, pp. 23-30, 1981.

[4] P. Zhao, S. Yang, and R. Yu, "Long-term changes in rainfall over eastern China and large-scale atmospheric circulation associated with recent global warming," Journal of Climate, vol. 23, no. 6, pp. 1544-1562, 2010.

[5] Y. J. Huang and X. P. Cui, "Moisture sources of torrential rainfall events in the Sichuan Basin of China during summers of 2009-13," Journal of Hydrometeorology, vol. 16, no. 4, pp. 1906-1917, 2015.

[6] T. Qian, P. Zhao, F. Zhang, and X. Bao, "Rainy-season precipitation over the Sichuan Basin and adjacent regions in Southwestern China," Monthly Weather Review, vol. 143, no. 1, pp. 383-394, 2015.

[7] X. Feng, C. Liu, R. Rasmussen, and G. Fan, "A 10-yr climatology of Tibetan plateau vortices with NCEP climate forecast system reanalysis," Journal of Applied Meteorology and Climatology, vol. 53, no. 1, pp. 34-46, 2014.

[8] B. Wang, "The development mechanism for Tibetan Plateau warm vortices," Journal of the Atmospheric Sciences, vol. 44, no. 20, pp. 2978-2994, 1987.

[9] S. H. Yu and W. L. Gao, "Large-scale conditions of Tibet Plateau vortex departure," Sciences in Cold and Arid Regions, vol. 1, pp. 559-569, 2009.

[10] S. Yu, W. Gao, J. Peng, and Y. Xiao, "Observational facts of sustained departure plateau vortexes," Journal of Meteorological Research, vol. 28, no. 2, pp. 296-307, 2014.

[11] S. Yu, W. Gao, D. Xiao, and J. Peng, "Observational facts regarding the joint activities of the Southwest vortex and plateau vortex after its departure from the Tibetan plateau," Advances in Atmospheric Sciences, vol. 33, no. 1, pp. 34-46, 2016. 
[12] Y. Chen, Y. Li, and T. Zhao, "Cause analysis on eastward movement of southwest China vortex and its induced heavy rainfall in South China," Advances in Meteorology, vol. 2015, Article ID 481735, 22 pages, 2015.

[13] Y. H. Kuo, L. S. Cheng, and R. A. Anthes, "Mesoscale analyses of the Sichuan flood catastrophe, 11-15 July 1981," Monthly Weather Review, vol. 114, pp. 1984-2003, 1986.

[14] Y.-H. Kuo, L. Cheng, and J.-W. Bao, "Numerical simulation of the 1981 Sichuan flood. Part I: evolution of a mesoscale Southwest vortex," Monthly Weather Review, vol. 116, no. 12, pp. 2481-2504, 1988.

[15] J. H. Lu, Generality of the Southwest vortex, China Meteorological Press, Beijing, China, 1986, in Chinese.

[16] A. Duan, R. Sun, and J. He, "Impact of surface sensible heating over the Tibetan plateau on the Western Pacific subtropical high: a land-air-sea interaction perspective," Advances in Atmospheric Sciences, vol. 34, no. 2, pp. 157-168, 2017.

[17] G. B. He and R. Shi, "Studies on dynamic and thermal characteristics of different shear lines over Tibetan plateau in summer," Plateau Meteorology, vol. 30, no. 3, pp. 568-575, 2011, in Chinese.

[18] Q. Z. Chen, Y. W. Huang, Q. W. Wang, and Z. M. Tan, "The statistical study of the southwest vortex during 1990-2004," Journal of Nanjing University (Natural Sciences), vol. 43, no. 6, pp. 633-642, 2007, in Chinese.

[19] J. Curio, R. Schiemann, K. I. Hodges, and A. G. Turner, "Climatology of Tibetan plateau vortices in reanalysis data and a high-resolution global climate model," Journal of Climate, vol. 32, no. 6, pp. 1933-1950, 2018.

[20] L. Li, R. Zhang, and M. Wen, "Diurnal variation in the occurrence frequency of the Tibetan plateau vortices," Meteorology and Atmospheric Physics, vol. 125, no. 3-4, pp. 135-144, 2014.

[21] Q. W. Wang and Z. M. Tan, "Multi-scale topographic control of southwest vortex formation in Tibetan plateau region in an idealized simulation," Journal of Geophysical Research: Atmospheres, vol. 119, no. 20, pp. 11543-11561, 2014.

[22] Z. Wang and K. Gao, "Sensitivity experiments of an eastwardmoving southwest vortex to initial perturbations," Advances in Atmospheric Sciences, vol. 20, no. 4, pp. 638-649, 2003.

[23] G.-X. Wu and S.-J. Chen, "The effect of mechanical forcing on the formation of a mesoscale vortex," Quarterly Journal of the Royal Meteorological Society, vol. 111, no. 470, pp. 1049-1070, 1985.

[24] X. Cheng, Y. Li, and L. Xu, "An analysis of an extreme rainstorm caused by the interaction of the Tibetan plateau vortex and the Southwest China vortex from an intensive observation," Meteorology and Atmospheric Physics, vol. 128, no. 3, pp. 373-399, 2016.

[25] S. Xiang, Y. Li, D. Li, and S. Yang, "An analysis of heavy precipitation caused by a retracing plateau vortex based on TRMM data," Meteorology and Atmospheric Physics, vol. 122, no. 1-2, pp. 33-45, 2013.

[26] Y. Chen, Y. Li, and D. Qi, "Analysis of the convective characteristics during the mutual evolution of an inverted trough/low vortex and its induced rainstorm over the northeastern Sichuan basin, China," Meteorology and Atmospheric Physics, pp. 1-19, 2018.

[27] Y. Chen, Y. Li, and L. Kang, “An index reflecting mesoscale vortex-vortex interaction and its diagnostic applications for rainstorm area," Atmospheric Science Letters, p. 902, 2019.

[28] G. Li and J. Deng, "Atmospheric water monitoring by using ground-based GPS during heavy rains produced by TPV and
SWV," Advances in Meteorology, vol. 2013, Article ID 793957 , 12 pages, 2013.

[29] B. Wang and I. Orlanski, "Study of a heavy rain vortex formed over the eastern flank of the Tibetan plateau," Monthly Weather Review, vol. 115, no. 7, pp. 1370-1393, 1987.

[30] S. Y. Tao, Rainstorms in China (in Chinese), Science Press, Beijing, China, 1980.

[31] Y. Luo, M. Wu, F. Ren, J. Li, and W.-K. Wong, "Synoptic situations of extreme hourly precipitation over China," Journal of Climate, vol. 29, no. 24, pp. 8703-8719, 2016.

[32] R. Yu and J. Li, "Hourly rainfall changes in response to surface air temperature over eastern contiguous China," Journal of Climate, vol. 25, no. 19, pp. 6851-6861, 2012.

[33] R. Yu, J. Li, W. Yuan, and H. Chen, "Changes in characteristics of late-summer precipitation over eastern China in the past 40 years revealed by hourly precipitation data," Journal of Climate, vol. 23, no. 12, pp. 3390-3396, 2010.

[34] P. Zhai, X. Zhang, H. Wan, and X. Pan, "Trends in total precipitation and frequency of daily precipitation extremes over China," Journal of Climate, vol. 18, no. 7, pp. 1096-1108, 2005.

[35] Q.-s. Chen and D. H. Bromwich, "An equivalent isobaric geopotential height and its application to synoptic analysis and a generalized $\omega$ equation in $\sigma$ coordinates," Monthly Weather Review, vol. 127, no. 2, pp. 145-172, 1999.

[36] K. E. Trenberth, "On the interpretation of the diagnostic quasi-geostrophic omega equation," Monthly Weather Review, vol. 106, no. 1, pp. 131-137, 1978.

[37] R. Davies-Jones, "On formulas for equivalent potential temperature," Monthly Weather Review, vol. 137, no. 9, pp. 3138-3147, 2009.

[38] H. Zong and Q. Zhang, "A new precipitation index for the spatiotemporal distribution of drought and flooding in the reaches of the Yangtze and Huaihe rivers and related characteristics of atmospheric circulation," Advances in Atmospheric Sciences, vol. 28, no. 2, 2011.

[39] W. D. Bonner, "Climatology of the low level jet," Monthly Weather Review, vol. 96, no. 12, pp. 833-850, 1968.

[40] H. B. Liu, L. J. Li, and B. Wang, "Low-level jets over Southeast China: the warm season climatology of the summer of 2003," Atmospheric and Oceanic Science Letters, vol. 5, no. 5, pp. 394-400, 2012.

[41] J.-L. Sortais, J.-P. Cammas, X. D. Yu, E. Richard, and R. Rosset, "A case study of coupling between low- and upperlevel jet-front systems: investigation of dynamical and diabatic processes," Monthly Weather Review, vol. 121, no. 8, pp. 2239-2253, 1993.

[42] L. W. Uccellini and D. R. Johnson, "The coupling of upper and lower tropospheric jet streaks and implications for the development of severe convective storms," Monthly Weather Review, vol. 107, no. 6, pp. 682-703, 1979.

[43] R. C. Miller, "Notes on analysis and severe storm forecasting procedures of the air force global weather central," Revision of Technical Report 200, Air Weather Service, Washington, DC, USA, 1972.

[44] J. E. Hales Jr., "On the relationship of $250 \mathrm{mb}$ positive vorticity advection and horizontal divergence to tornado and severe thunderstorm development," in Proceedings of the Preprints of Eleventh Conference of Severe Local Storm, pp. 28-31, Kansas, MO, USA, October 1979.

[45] R. A. Maddox and C. A. Doswell, "An examination of jet stream configurations, $500 \mathrm{mb}$ vorticity advection and low-level thermal advection patterns during extended periods of intense convection," Monthly Weather Review, vol. 110, no. 3, pp. 184-197, 1982. 
[46] J. Molinari, D. M. Romps, D. Vollaro, and L. Nguyen, "CAPE in tropical cyclones," Journal of the Atmospheric Sciences, vol. 69, no. 8, pp. 2452-2463, 2012.

[47] N. T. Atkins, R. M. Wakimoto, and C. L. Ziegler, "Observations of the finescale structure of a dryline during VORTEX 95," Monthly Weather Review, vol. 126, no. 3, pp. 525-550, 1998.

[48] R. S. Duell and M. S. Van Den Broeke, "Climatology, synoptic conditions, and misanalyses of Mississippi river valley drylines," Monthly Weather Review, vol. 144, no. 3, pp. 927-943, 2016. 

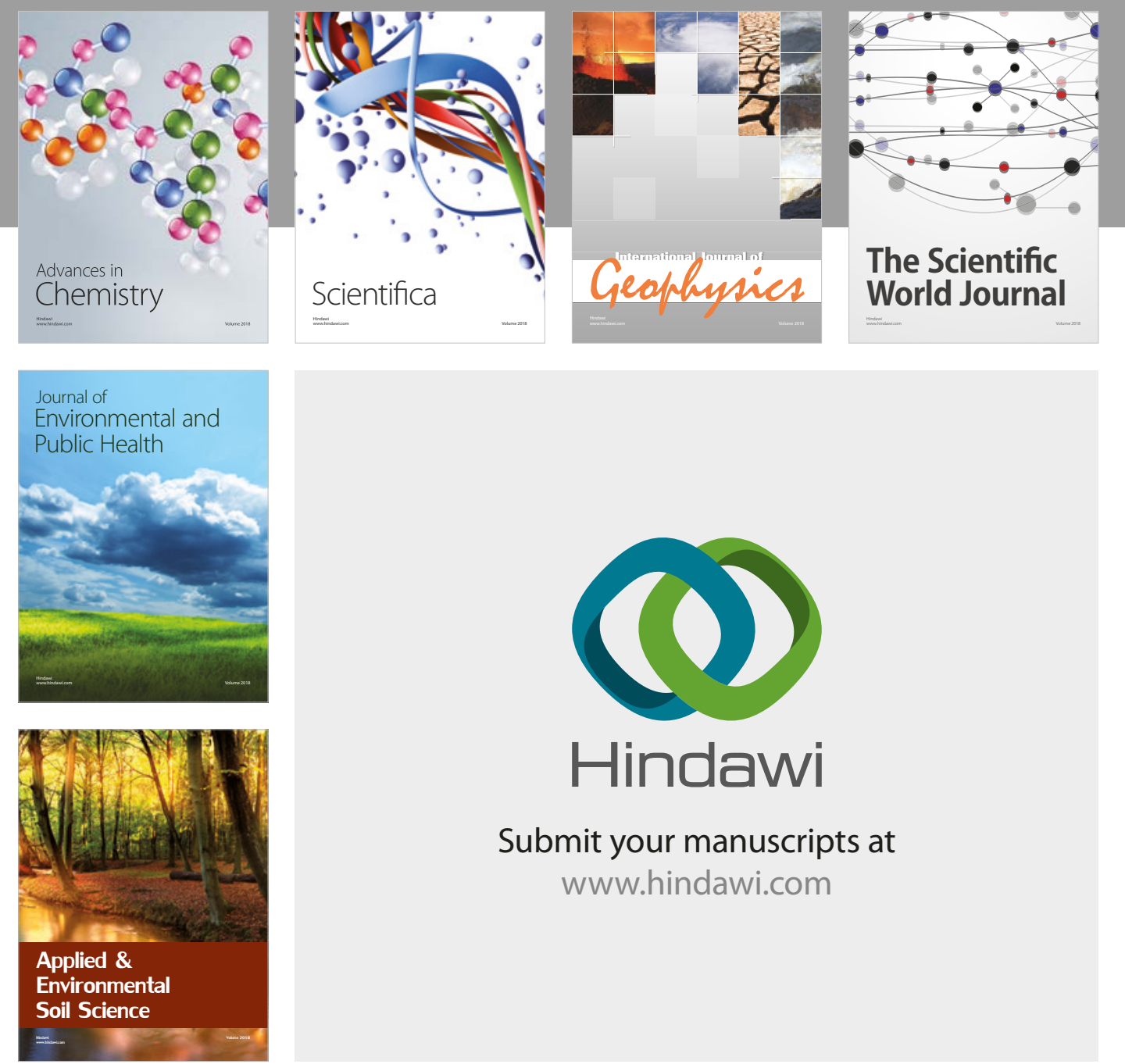

The Scientific

\section{World Journal}
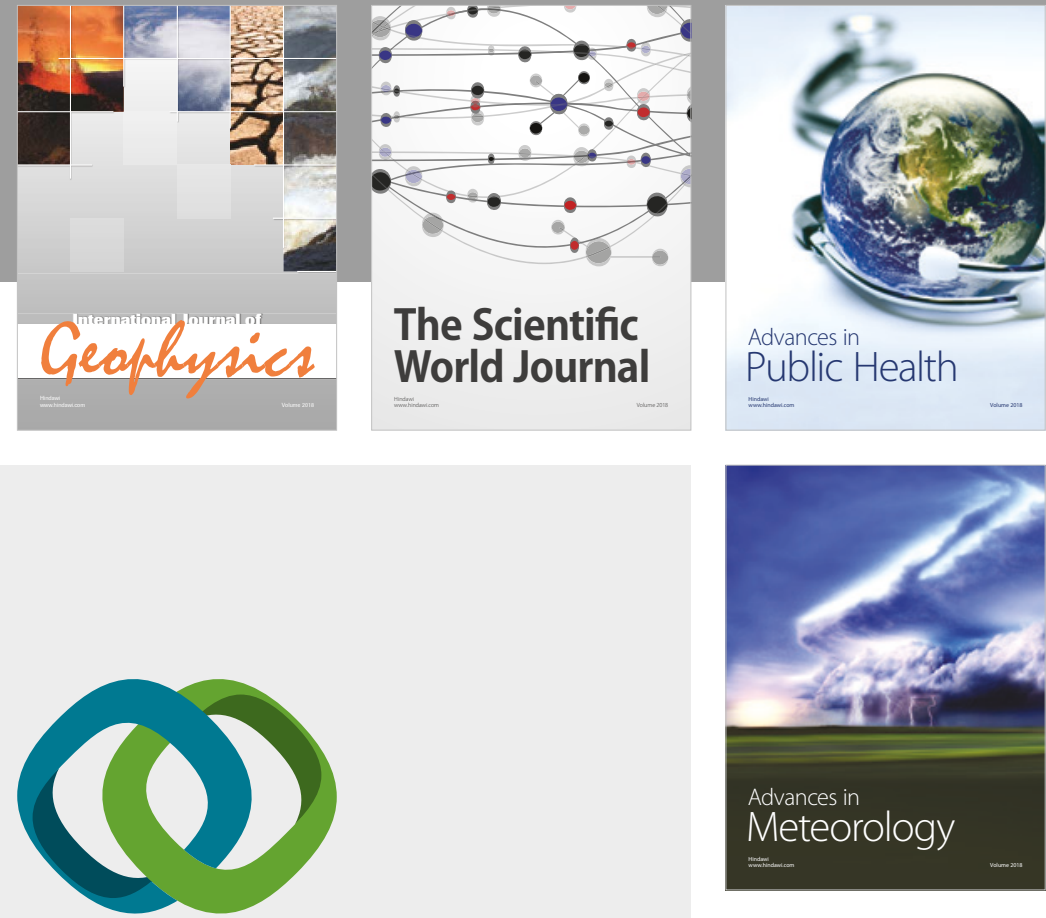

Advan

Public Health

\section{Hindawi}

Submit your manuscripts at

www.hindawi.com
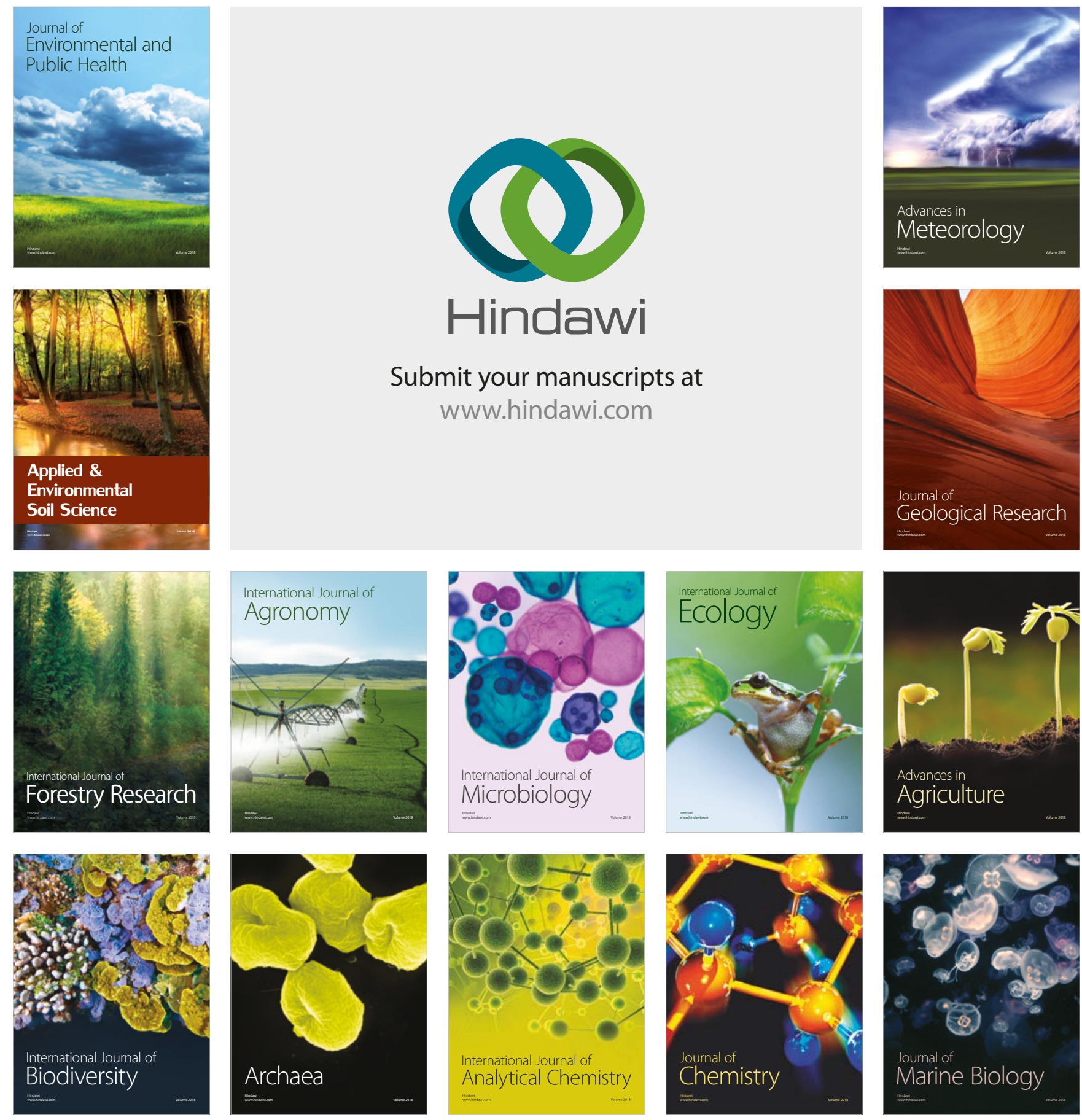